\title{
Biología del grupo prehistórico de Pisagua, costa norte de Chile $^{1}$
}

\author{
José A. Cocilovo ${ }^{2}$, Silvia Quevedo ${ }^{3}$, Hector H. Varela ${ }^{2}$, Silvia Valdano ${ }^{2}$ y Mario Castro ${ }^{3}$
}

\section{RESUMEN}

En la costa norte de Chile y en las proximidades de la actual localidad de Pisagua, vivió un grupo prehistórico de pescadores desde el $300 \mathrm{AC}$ hasta el 1.450 DC. Sus restos, actualmente conservados en el Museo Nacional de Historia Natural (Santiago Chile), fueron exhumados por Max Uhle en 1913 en cuatro cementerios correspondientes a tres etapas del desarrollo agroalfarero del Norte de Chile (Formativo, Tiwanaku y Desarrollo Regional). El objetivo del presente trabajo es el estudio de la estructura de la población a partir del análisis de la distribución de rasgos fenotípicos. Bajo el supuesto de distribución al azar de efectos no genéticos, dicha estructura refleja cambios genéticos que dependen de la acción de factores microevolutivos asociados con eventos históricos producidos a nivel regional y local. Se relevó un conjunto de rasgos morfológicos (atributos y variables continuas) en un total de 120 individuos y se analizó la distribución de estos rasgos de acuerdo con el sexo, la edad, la deformación artificial y el cementerio de origen, aplicando técnicas de análisis estadístico uni y multivariadas. Esta informa-

Trabajo realizado con subsidios de FONDECYT (Chile, Proyecto 348, 1174/83), SUBCYT (Argentina, Resol. 417/83, 150/85) y UNRC (programa 477 I 83/84/85).

Departamento de Ciencias Naturales, Facultad de Ciencias Exactas, Físico-Químicas y Naturales, Universidad Nacional de Río Cuarto y CONICET, Campus, 5800 RIO CUARTO, Argentina, jcocilovo@exa.unre.edu.ar

3 Museo Nacional de Historia Natural, Casilla 787. Santiago, Chile. ción es completada con observaciones sobre la incidencia de algunas patologías, el estado de la dentición y las características del perfil demográfico. Se establecen sus relaciones biológicas con otros grupos próximos del Norte de Chile y se realizan inferencias sobre el origen probable de la población local.

\section{ABSTRACT}

On the northern coast of Chile and near of what is today the city of Pisagua, a group of prehistoric fishermen lived from $300 \mathrm{AC}$ to $1450 \mathrm{DC}$. Their remains, now preserved at the National Museum of Natural History, were exhumed by Max Uhle in 1913 in four burial sites corresponding to three stages of the agriculturist development of Northern Chile. The aim of the present work is the study of the population structure from the analysis of the phenotypic traits distribution. On the assumption of random distribution of non genetic effects, such structure reflects genetic changes which depend on the activity of microevolutionary factors associated with historical events which took place at a local and regional level. The achievement of the aims required the acquisition of the set of morphological traits (attributes and continuous variables) in 120 individuals. The study of the local biology was carried out through the analysis of the distribution of such features in relations to sex, age, artificial deformation and source cemetery. Uni and multivariate techniques were applied. This was completed with observation on the incidence of certain pathologies and dentition state, and characteristics of the demographic profile. Its biological relations with other nearly groups in northern Chile are established, and 
inferences are realized about local population origin.

\section{Introducción}

La reconstrucción de las características biológicas de las poblaciones humanas prehistóricas involucra la recuperación de información, a partir de una muestra de restos óseos, sobre el dimorfismo sexual, la variación etaria, el efecto de la deformación artificial, la incidencias de patologías, la nutrición y la demografía. Esta información permite evaluar de una manera más realista la diferenciación geográfica de los grupos cuando, a través del estudio de sus relaciones y afinidades biológicas, se intenta una explicación probable sobre los factores determinantes del proceso microevolutivo que operó en una determinada región. Trabajos de esta naturaleza, no abundan en el área andina centro meridional y particularmente en el norte de Chile. Aportes parciales pueden mencionarse para la serie de Morro de Arica exhumada por Max Uhle en 1912 (Cocilovo et al., 1982; Mendonça et al., 1983, 1986; Mendonça y Di Rienzo, 1981-82; Martino et al., 1991; Martino y Cocilovo, 1988, etc), derivados del desarrollo desde 1978 del proyecto conjunto "Microevolución en Poblaciones del Area Andina Meridional" entre especialistas argentinos y chilenos.

El presente informe se refiere a los principales resultados obtenidos durante el estudio de los materiales de la colección Max Uhle de la localidad de Pisagua, con la finalidad de brindar una contribución al conocimiento de la biología del grupo en el sentido antes indicado. Esto incluye además, la investigación de la estructura de la muestra según su distribución en cuatro cementerios posiblemente pertenecientes a distintos momentos del desarrollo cultural del norte de Chile y de sus relaciones con otras localidades. Un tratamiento más completo del tema se encuentra en Cocilovo (1995).

Para la zona de Pisagua, la arqueología hoy concibe un sistema de desarrollo cultural continuo con dataciones desde el VIII milenio AC, hasta la conquista española, desde Tiliviche (7810 $\pm 4110 \mathrm{AC})$, Punta Pichalo I ( $4206 \pm 220$ AC), Punta Pichalo II (3666 \pm 145 AC), Chinchorro (2930 $\pm 320 \mathrm{AC})$, Faldas del Morro (0-700 DC), Pichalo III (700 DC),
Tiwanaku (700 - 1000 DC), Pichalo IV (1000 DC), San Miguel (1200 DC), Gentilar ( 1350 DC) e Inca (1450 DC), hasta el colapso de la sociedad indígena en 1550 (Nuñez, 1965 y 1980). El material exhumado por Uhle en Pisagua cubre el registro desde el llamado complejo de Faldas El Morro hasta el siglo XIV de nuestra era, lapso durante el cual se espera, a partir de los datos culturales, una activa interacción entre la sociedad local y las poblaciones de otras subáreas y de otras regiones más alejadas.

El paisaje de Pisagua participa de las características generales vinculadas con el Desierto de Atacama en la costa árida, en la cual los principales factores limitantes para el desarrollo de la población son la disponibilidad de agua, de alimentos y de combustible. El clima es desértico con nublados abundantes (BWn) con una alta humedad relativa, gran frecuencia de días nublados, escasas precipitaciones y régimen térmico homogéneo; los vientos dominantes son de componente sur y sudoeste (Errazuriz et al., 1987; Nuñez y Varela, 1967-68). El principal accidente geográfico lo constituye la Punta Pichalo (19०36'30'latitud y $70^{\circ} 19^{\prime}$ de longitud W). El relieve es abrupto y no existe una terraza de abrasión verdadera, tampoco hay una desembocadura de río propiamente dicha, sino un poco más al norte cerca de Pisagua Viejo. Comenzando casi en el borde del agua, el terreno sube en una marcada pendiente por 335 $\mathrm{m}$, al cabo de la cual continúa por $450 \mathrm{~m}$ más hasta el nivel general de la meseta costera. Los yacimientos se encuentran localizados en promontorios rocosos o eminencias destacadas que ofrecen reparo para el oleaje y permiten, a la vez, la práctica de la pesca como fuente principal de recursos. Este modo de vida parece haber perdurado aún después de la introducción de la agricultura cuya producción no debe haber alcanzado para inducir un cambio substancial en el tipo de subsistencia (Bird, 1943 y 1946).

Próxima hacia el norte, se desarrolla la quebrada de Tana o Camiña que se continúa con la de Tiliviche y la de Jazpampa, constituyendo una vía probable de comunicación que en tiempos preagrícolas permitió integrar el aprovechamiento de los recursos de distintas regiones, en un mecanismo de transhumancia, entre Pisagua y la llamada Pampa del Tamarugal, con la existencia pro- 
bada de campamentos intermedios como: Saya, Quiuña Bajo, Tiliviche y Aragón (Nuñez et al., 1976). Seguramente, a este circuito no debió estar ajena la zona altiplánica, que en épocas posteriores pudo haber aportado recursos agropecuarios propios como la quinua y la papa, que hasta hoy se manejan con una tecnología similar por ejemplo en Isluga (Lanino Rozas, 1977; Albornoz Acosta, 1977).

El conocimiento de las interacciones biosociales implica además del aporte de la arqueología, la incorporación de los datos obtenidos por medio de los estudios de relaciones biológicas entre los distintos grupos. Investigaciones anteriores permitieron comprender la variación geográfica de la morfología de los grupos prehistóricos desde Perú hasta Tierra del Fuego, y su correlación con la distribución espacial (Cocilovo, 1981; Cocilovo y Di Rienzo, 1985). Casi simultáneamente se encararon trabajos para estudiar la variación cronológica de la morfología craneana desde el punto de vista métrico y no métrico en el Valle de Azapa (Norte de Chile), cuyos resultados permitieron verificar una alta correlación entre las distancias $D^{2}$ y las diferencias cronológicas entre las muestras, habiéndose postulado la acción de migraciones de amplio rango sobre la región (Rothhammer et al., 1981, 1982 y 1984). Con posterioridad se demostró la influencia en esa región de poblaciones altiplánicas (Rothhammer et al., 1983) y amazónicas (Rivera y Rothhammer, 1986), sentándose las bases para explicar el poblamiento del Norte de Chile a partir de cinco corrientes migratorias (Rothhammer et al., 1984; Rothhammer et al., 1986).

Empleando un conjunto de muestras con cronologías conocidas y aproximadas de Perú, Bolivia, N.O. Argentino y Norte de Chile se preparó el primer cuadro de desarrollo biológico de los grupos prehistóricos del área Centro Sur Andina y se proporcionaron las hipótesis más probables sobre el origen de las poblaciones de varias localidades. La estructura básica estuvo fundada en los desarrollos del Valle de Azapa, de Pisagua y de San Pedro de Atacama. Los principales resultados indican la conformación de dos grandes conglomerados separados por la cordillera andina: uno en el Norte de Chile y otro en el N.O. Argentino, contando cada uno de ellos con un conjunto de pobla- ciones locales que desde el Período Arcaico se fueron paulatinamente diferenciando desde el punto de vista biológico en áreas geográficas y en biotopos específicos, a pesar de las comprobadas interacciones culturales entre ellas (Cocilovo y Rothhammer, 1990).

Por razones técnicas, los trabajos antes citados se realizaron empleando un conjunto de nueve variables craneométricas. En cada muestra la influencia de los factores de variación interna como el sexo, la edad y la deformación artificial en la posición del vector de medias no fue adecuadamente resuelto por el escaso número de observaciones disponibles en varias series y por desconocimiento del verdadero efecto sobre la estimación de las distancias $D^{2}$. Las experiencias realizadas aquí intentan resolver este problema mediante una evaluación previa de tales factores en la expresión de las características métricas y la posterior reducción de su efecto por la aplicación de una técnica numérica que permite trabajar con los valores residuales, descontando la variación producida por cada uno de ellos.

\section{Material y métodos}

De acuerdo con la información proporcionada en los catálogos del Museo Nacional de Historia Natural la colección de Pisagua fue recuperada por Max Uhle en los meses de mayo, setiembre y noviembre de 1913, a partir de cuatro cementerios designados con las letras "H", "d", "c" y "A", ubicados en los alrededores del poblado actual y de Punta Pichalo (figura 1). Siguiendo los trabajos de Uhle (1913 y 1919) y las propuestas de Nuñez $(1965,1968$ y 1978) es posible asignar cada cementerio, con alguna aproximación, a un momento determinado del desarrollo cultural del Norte de Chile. Sobre los depósitos precerámicos se dispone la fase de ocupación Pichalo III (200 300 DC) con la incorporación efectiva de recursos agrícolas, cerámica, el uso de grandes turbantes e inhumaciones en posición genuflexa en grandes canastos, equivalentes al inventario del sitio "d" $y$ al Ilamado complejo Faldas del Morro de Arica (Formativo). Luego, con rasgos Tiwanaku principalmente en los textiles, se habría formado una nueva ocupación (400 al 1000 DC) atestiguada por los restos hallados en los cementerios " $\mathrm{c}$ " $\mathrm{y}$ "A". El desarrollo postiwanaku (Desarrollo Regional) 
se correspondería con los materiales del cementerio "H" y a la fase Pichalo IV (1000 al 1430 DC), con contactos evidentes con la cultura Arica (Figura 1).
Aunque la citada colección está compuesta por cerca de un centenar de restos óseos, 60 cuerpos momificados y abundante material cultural exhumados en cuatro cementerios próximos a la

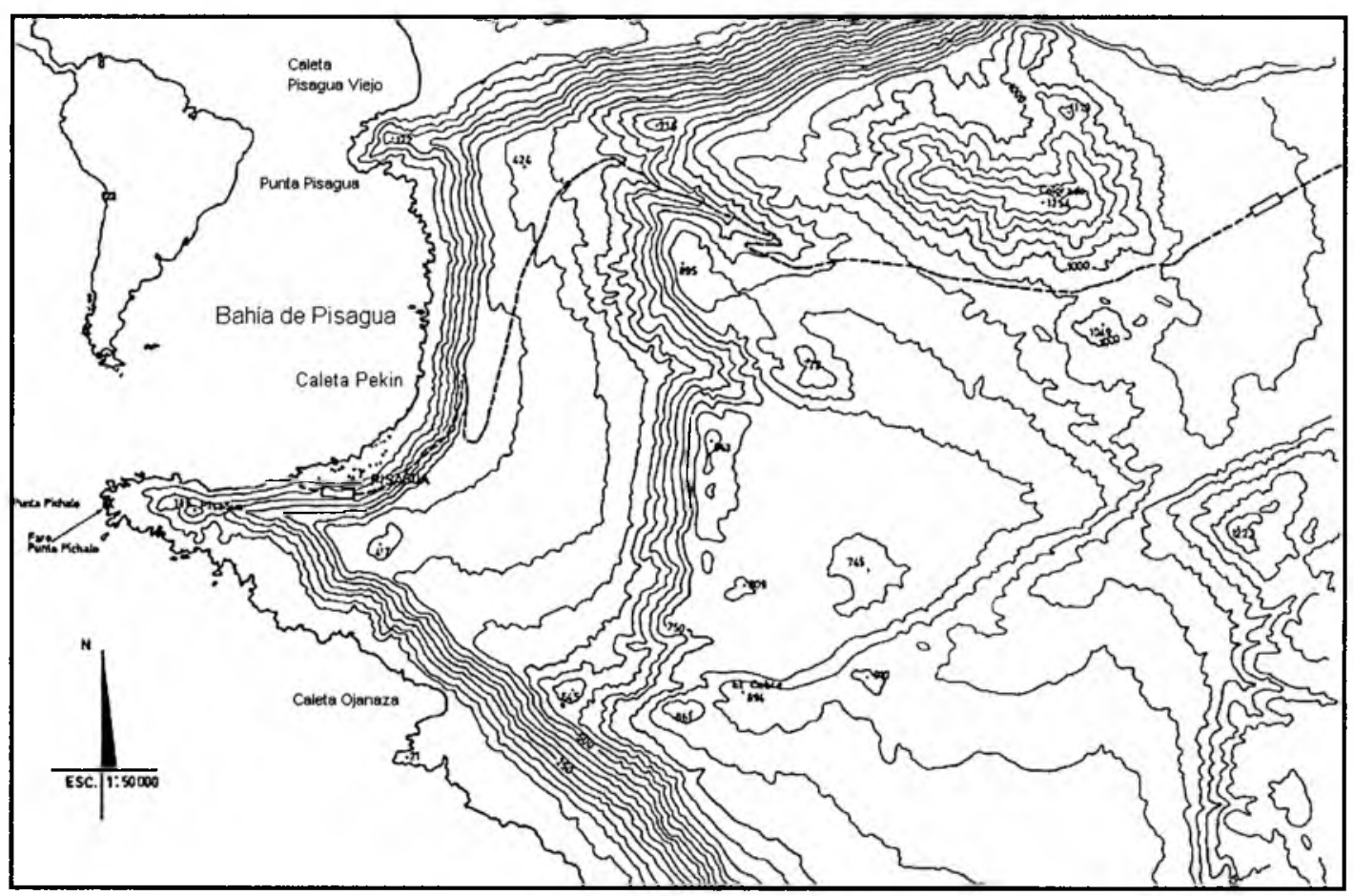

Figura 1:

Ubicación de la localidad de Pisagua (costa norte de Chile) en la cual se obtuvieron los materiales empleados en el presente trabajo.

Tabla 1:

Pisagua. Composición y estructura de la muestra por cementerio, sexo y edad.

\begin{tabular}{|c|c|c|c|c|c|c|c|c|c|c|c|c|c|c|c|c|}
\hline COMPONENTE & \multicolumn{3}{|c|}{$\mathrm{D}$} & \multicolumn{3}{|c|}{$\mathrm{C}$} & \multicolumn{3}{|c|}{$\bar{A}$} & \multicolumn{3}{|c|}{$\mathrm{H}$} & \multicolumn{3}{|c|}{ Total } & Total \\
\hline EDAD $\quad$ SEXO & & $\mathrm{F}$ & I & $\mathrm{M}$ & $\mathrm{F}$ & & $\bar{M}$ & $\bar{F}$ & I & $\bar{M}$ & $\mathrm{~F}$ & I & $\bar{M}$ & $\bar{F}$ & I & General \\
\hline NINO & & & 1 & & & & & & 6 & & & 3 & & 1 & 14 & 15 \\
\hline SUB ADULTO & & 2 & & & & & & 2 & & & & 1 & 1 & 6 & 2 & 9 \\
\hline ADULTO JOVEN & 1 & 1 & & & & & & & & & 1 & & 2 & 2 & & 4 \\
\hline ADULTO & & 5 & & & & & 1 & 8 & & 7 & 1 & 1 & 22 & 16 & 1 & 39 \\
\hline AD. MADURO & & 5 & & & & & 3 & 5 & & 2 & 2 & 2 & 16 & 15 & 2 & 33 \\
\hline AD. MAD. AVA. & 2 & & & & & & 3 & 3 & & 9 & & & 16 & 4 & & 20 \\
\hline Sub Total & & 14 & 1 & 1 & & & 7 & 18 & 6 & 18 & 4 & 7 & 57 & 44 & 19 & \\
\hline Total & & & & & 2 & & & 31 & & & 29 & & & & & 120 \\
\hline
\end{tabular}

$\mathrm{M}=$ Masculinos, $\mathrm{F}=$ Femeninos, $\mathrm{I}=$ Indeterminados $; \mathrm{D}=$ Protonazca, $\mathrm{C}=$ Tiw anaku, $\mathrm{A}=$ Tiwanaku-Atacameño y $\mathrm{H}=$ Atacameño. 
localidad homónima, la muestra que en realidad fue trabajada se consigna a continuación (Tabla $1)$.

Los restos óseos disponibles para su estudio están representados casi exclusivamente por cabezas y cráneos, el material postcraneano es sumamente escaso y no fue considerado. Se observa una proporción mayor de individuos masculinos que de femeninos; los indeterminados son ejemplares infantiles. Son más frecuentes las piezas que presentan algún tipo de deformación artificial, y entre éstos predominan los tabulares; los indeterminados con respecto a este atributo son restos muy fragmentados.

Las tareas de relevamiento fueron realizadas en el Museo Nacional de Historia Natural de Santiago (Chile), las cuales incluyeron la observación sistemática de un conjunto de rasgos: atributos mayores (morfoscopía gruesa), atributos menores (rasgos no métricos) y variables métricas. La información recuperada fue primeramente consignada en forma estandarizada en fichas especiales y controlada por más de un observador. El relevamiento de 29 rasgos anatómicos gruesos, en las cinco normas craneales, fue llevado a cabo de acuerdo con Comas (1966), Bass (1971), Brothwell (1981), Stewart (1979), Hrdliçka (1947) y Anderson (1962), complementadas con Testut (1921), Bórmida (1953/54 y 1961/63), Neuman (1942), Weiss (1961), Dembo e Imbelioni (1938) y Ferembach, Schwidetzky y Sloutkal (1979). La observación de 35 rasgos no métricos se hizo de acuerdo con el trabajo de Castro y Quevedo (198384). La determinación del sexo, de la edad y de la deformación artificial fue encarada según Genovés (1962), Stewart (1979), Imbelloni (1925), Dembo e Imbelloni (1938), Neuman (1942), Weiss (1961 y 1962), y particularmente Ferembach, Schwidetzky y Sloukal (1979). Las mediciones de 53 variables fueron realizadas de acuerdo con las recomendaciones de la Convención Internacional de Mónaco (1906, en Comas 1966), Wilder (1920) y Bass (1971).

La muestra disponible fue clasificada por clases de edad, sexo, deformación y cementerio, procediéndose con posterioridad al estudio de la distribución de las distintas variables de acuerdo con la variación etaria, el dimorfismo sexual, la deformación artificial y los componentes funera- rios. Como no es posible suponer ausencia de interacción entre los citados factores, los diseños experimentales empleados fueron preparados para superar este inconveniente. En el caso de los atributos se tuvo en cuenta poner a prueba previamente la independencia entre estos factores (sexo $x$ edad, sexo $x$ deformación, sexo $x$ cementerio, edad $\mathrm{x}$ deformación $\mathrm{y}$ edad $\mathrm{x}$ cementerio $\mathrm{y}$ deformación $x$ cementerio) antes de hacer inferencias sobre la distribución de una determinada característica en función de ellos. En todos los casos, las variantes no métricas fueron evaluadas como presencia y ausencia y para los atributos mayores o gruesos, se siguieron las categorías que se especifican en la tabla del Apéndice 1. Con respecto a las variables métricas se procedió a realizar la dócima de cada efecto por separado eliminando antes la variación producida por los otros, es decir, trabajando con los valores residuales (Sokal y Rohlf, 1979).

Las dócimas de hipótesis referidas a proporciones para las experiencias con caracteres morfológicos gruesos y rasgos no métricos fueron realizadas empleando la distribución $\mathrm{Chi}^{2}$ para tablas de contingencia $2 \times 2$ y R x C (Snedecor y Cochran, 1984; Lison, 1976). La evaluación de las diferencias entre valores medios para edad, dimorfismo sexual, deformación y cementerios fue realizada por medio del análisis de la varianza para un criterio y dos o más niveles. Previamente fueron controlados los supuestos de distribución normal y de homocedasticidad por las estadísticas $W_{1}$ $\mathrm{y} \mathrm{W}_{2}$ y sus correlativas $\mathbf{W}$ y $\mathbf{u}$ para la asimetría y la curtosis, y M' de Barttlet (Rao,1952:218-219; Schapiro y Wilk, 1965; David et al., 1954; Bliss, 1967:239-241; Sokal y Rohlf, 1979; Scheffé, 1959). También, las diferencias entre cementerios fueron evaluadas empleando técnicas de estadística multivariada.

La reconstrucción de la estructura paleodemográfica del grupo de Pisagua fue encarada siguiendo las recomendaciones de Ubelaker (1974) para el cálculo de la tabla de vida, considerando la muestra total como derivada de una única población.

El estudio de las relaciones y afinidades biológicas entre Pisagua y otras series del Norte de Chile (Morro de Arica, San Pedro de Atacama y Calama) fue desarrollado analizando las diferencias 
entre las localidades. Bajo el supuesto de distribución normal multivariada e igualdad de matrices de dispersión, las diferencias entre los vectores medios de $\mathbf{k}$ muestras $\mathbf{p}$ variadas empleando variables métricas pueden ser docimadas mediante la estadística Lambda de Wilk (Seber, 1984; Rao, 1952) y su aproximación basada en la distribución F. Para el caso particular cuando $k=2$ (Rao, 1952; Seber, 1984) las diferencias entre pares de grupos pueden ser evaluadas por el valor $D^{2}$ transformado en un cociente entre varianzas $F$ con $p y$ $m$ grados de libertad. Partiendo de un conjunto de 36 mediciones y 291 observaciones corregidas para edad, sexo y deformación artificial, se aplicó un procedimiento de selección multivariado paso a paso que permitió obtener un subconjunto de 13 variables elegidas como las de mayor poder discriminante. Con estas mediciones se realizaron los cálculos del análisis discriminante canónico.

Con la denominación de Morro de Arica definimos una muestra osteológica derivada de la colección del Museo Nacional de Historia Natural (Santiago, Chile) obtenida por Max Uhle en 1912 en cementerios de la Pampa de Chinchorro y en los faldeos del Morro de la ciudad de Arica. Corresponde a una fase del desarrollo cultural preagroalfarero contemporánea con el llamado complejo Chinchorro (3000 AC), resultante de la actividad de una comunidad de hábitos costeros con una economía cazadora y pescadora (Dauelsberg, 1974; Rivera, 1975; Mostny, 1977). En esta oportunidad hemos empleado 44 ejemplares de ambos sexos, de edad adulto y maduro, deformados circularmente y no deformados.

San Pedro de Atacama constituye una serie de 114 ejemplares de la colección del Museo de Arqueología de la localidad homónima, integrada como la anterior con piezas masculinas y femeninas, adultos, maduros y seniles, y cráneos deformados (tabulares erectos y oblicuos) y no deformados. Estos materiales corresponden a los siguientes períodos: San Pedro I (500 AC-300 DC), San Pedro II (300 DC-900 DC), San Pedro IIIa (900 DC-1300 DC) y San Pedro IIIb (1300 DC-1536 DC) de acuerdo con la observaciones de Costa Junqueira (1985) sobre la periodificación original de Orellana (1963a y b). En este trabajo, por razones técnicas relacionadas con la cantidad de observaciones por submuestra, se consideraron jun- tos los materiales de algunas fases en el esquema que sigue: San Pedro I incluye materiales de I y I/ II, San Pedro II de II y II/III, San Pedro III de III (IIIa) y San Pedro IV de IV (IIIb). La presente serie fue obtenida en los cementerios Toconao Oriental, Quitor 1, 2, 5, 6, y 8, Coyo Oriental, Yaye $1,2,3, y 4$, sumando en total 118 ejemplares. Un estudio completo sobre esta colección fue realizado por Varela (1997).

Con el nombre de Calama designamos una muestra de 66 cráneos obtenida en 1912 por Max Uhle en el cementerio de Chunchurí próximo a la localidad citada, también formada por ejemplares de ambos sexos, adultos, maduros y seniles, deformados artificialmente y no deformados. Esta localidad corresponde al yacimiento Dupont- 1 del período de Desarrollo Regional postiwanaku datado en $1490 \pm 100$ (Nuñez, 1965).

\section{Resultados}

Tal como fueron diseñadas las distintas experiencias realizadas en el presente estudio, se exponen los siguientes resultados con referencia al dimorfismo sexual y a la variación etaria, a la deformación artificial y a la diferenciación por cementerios, a la paleodemografía y a las relaciones con otros grupos. Un estudio más completo se encuentra en Cocilovo (1995).

\section{Dimorfismo Sexual}

En la tabla 2 se consignan los resultados de las dócimas de hipótesis de independencia referidas a los atributos mayores. No hemos obtenido pruebas suficientes como para demostrar una asociación entre el sexo, la edad y la deformación artificial de las piezas. También parece ser independiente de este carácter el grado de conservación, el desgaste dentario, la prominencia del vertex y del occipucio, el perfil craneano, los arcos cigomáticos (cripto y fenocigia) y la forma del foramen magnun. En cambio, se comprueba claramente el dimorfismo sexual con relación al tamaño y al peso de los cráneos. La prominencia glabelar, el surco retroglabelar, los arcos superciliares y los relieves óseos son más marcados en los cráneos masculinos y en los femeninos predominan las caras angostas, los huesos nasales son menos pronunciados, las aberturas y las espi- 
Tabla 2:

Atributos Mayores, valores $C h i^{2}$ de las pruebas de independencia para sexo, edad, deformación artificial y variación entre cementerios

\begin{tabular}{|c|c|c|c|c|c|c|c|}
\hline VARIABLE & SEXO & & $\overline{\text { EDAD }}$ & & DEFORMAC ION & CEMENTER IO & G.I. \\
\hline SEXO & & & & & & $11,968^{* *}$ & \\
\hline EDAD & 7,306 & & & & & 9,402 & \\
\hline DEFORMACION & $3,07 \mathrm{I}$ & & 13,419 & & & $35,581^{\text {*** }}$ & \\
\hline CONSERVACION & 0,489 & & 5,436 & & 2,795 & 1,738 & \\
\hline TAMANO DEL CRANEO & & 2 & $35.306^{* * *}$ & 6 & $16,648^{*}$ & $15,040^{*}$ & \\
\hline PESO DEL CRANEO & & 2 & $23,885^{* * k}$ & 6 & $17,499^{*}$ & 11,798 & \\
\hline RELIEVES OSEOS & & 2 & $30,284^{* * *}$ & 6 & $16,084^{*}$ & $21,387^{* * *}$ & \\
\hline FORMA DE LA CARA & & 1 & $1 \overline{5.403^{* *}}$ & 3 & 0,826 & $11,129^{*}$ & \\
\hline$\overline{\text { GLABELA }}$ & & 1 & 7,438 & & $17,914 * *$ & $12,069 * *$ & \\
\hline SUTURA METOPICA & $7,182^{*}$ & & $10.152^{*}$ & & 6,483 & 4,312 & \\
\hline ARCOS SU PERCILIARES & & 2 & $42,433^{* *}$ & 6 & 2,380 & $13,018^{*}$ & \\
\hline FORMA DE LAS ORBITAS & $5,620 *$ & & $8, \overline{798}$ & & 3,194 & 1,292 & \\
\hline ABERTURAS NASALES & $7.855^{*}$ & & $14.095^{*}$ & & 7.527 & 3,225 & \\
\hline ESPINA NASAL & & $\mathrm{I}$ & $11,539 *$ & & 2,955 & 2,092 & \\
\hline TAMANO HUESO MALAR & & 2 & $35.008 \%$ & 6 & 8,630 & 11,016 & \\
\hline DESGASTE DENTARIO & 3,007 & & $44.426^{* *}$ & 6 & 15,188 & 6,648 & \\
\hline VERTEX & 5,142 & & 5,241 & & $30,328^{* *}$ & 3.203 & \\
\hline INCLINACION FRONTAL & $9.936^{* *}$ & & $14.690^{*}$ & & $27.787 * *$ & $15,026 *$ & \\
\hline SURCO RETROGLABELAR & & 1 & $13,075^{* * *}$ & 3 & 0,878 & 3,630 & \\
\hline PROMINEN. HUESOS NASALES & $11,000^{* *}$ & 1 & $18,200^{* *}$ & 3 & 4,131 & 5,055 & \\
\hline ARCOS CIGOMATICOS & & 2 & $45,958^{* * *}$ & 6 & 6,953 & 8.008 & \\
\hline CREST A SUPRAMASTOIDEA & $8,969 * *$ & & $17,862^{*}$ & & 4,465 & 4,595 & \\
\hline OCCIPUCIO & 0,323 & & 0,553 & & 3,794 & 0,698 & \\
\hline APOFISIS MASTOIDEA & & 2 & $42,525 * \%$ & 6 & 11,386 & 10,361 & \\
\hline ALTURA DE LA BOVEDA & $9,688^{* *}$ & & 1,947 & & $28,248^{* * *}$ & $15,620^{*}$ & \\
\hline RELIEVE PLANO NUCAL & & 2 & $21,741 * *$ & 6 & $17,712^{\%}$ & 8,704 & \\
\hline PROTUB.OCCIPITAL EXTERNA & $20,639 * *$ & 2 & 7,528 & & $20,708^{* * *}$ & 6,956 & \\
\hline PERFIL CRANEANO & 3,028 & & 14,745 & & $38.516^{* * *}$ & $21.040^{*}$ & \\
\hline ARCOS CIGOMATICOS & 1,489 & & $18,521^{* *}$ & 3 & $12,872^{*}$ & 0,729 & \\
\hline PLAGIOCEFALIA & $8.028 * *$ & & 3.402 & & $19.378 * *$ & 7,690 & \\
\hline FORMA FORAMEM MAGNUN & 0.914 & & 1,744 & & 0.428 & $10,493^{*}$ & \\
\hline DEPRESION ESFENOIDAL & & 2 & 7.922 & & 5,952 & 10,994 & \\
\hline TAMANO FOSAS GLENOIDEAS & $11,988^{* *}$ & 2 & $33,698 * *$ & 6 & 7,513 & 5,388 & \\
\hline
\end{tabular}

$* \mathrm{y} * *$ rechazos de la hipótes is nula a los niveles del 0,05 y 0,01 de probabilidad

nas nasales son pequeñas y la sutura metópica es más escasa. En los masculinos se destaca la cresta supramastoidea así como la apófisis mastoidea, el hueso malar es más robusto y el frontal más inclinado, las bóvedas son más altas y las líneas nucales y la cresta occipital aparecen más marcadas.

En la tabla 4 se exponen los resultados de la prueba $F$ sobre la igualdad de los valores medios de rasgos métricos entre uno y otro sexo. El supuesto de distribución normal se cumple en la mayoría de las variables, sin embargo hay algunos casos de curtosis y de asimetría marcada (altura sinfisiana y altura del cuerpo mandibular en femeninos). Se verificaron varianzas desiguales al nivel del 0,05 en el caso de la anchura bimastoidea y biastérica, de la longitud maxilo alveolar y de la altura del cuerpo mandibular. Por estas razo- nes, deben ser tomados con precaución u omitidos los resultados de las dócimas para diferencias entre sexos realizadas con estas características. Con respecto al resto hay 37 mediciones que se manifiestan diferentes entre masculinos y femeninos. Aquellos se caracterizan por alcanzar mayores dimensiones en la longitud y altura de la bóveda, en la anchura de la base craneal, en las dimensiones generales de la cara y en la altura de la nariz, en las dimensiones del foramen magnun, la capacidad craneana, en las curvas horizontal, nasion bregma y bregma lambda, y en el maxilar inferior.

En la tabla 3 figuran los valores de las dócimas de independencia para atributos menores (rasgos no métricos). En este ensayo no hemos obtenido pruebas suficientes para demostrar asociación entre el sexo de las piezas y la distribución de la mayoría 
Tabla 3:

Atributos menores, valores $\mathrm{Ch}^{2}$ para las dócimas de independencia referidas al sexo, la edad, la deformación artificial y el cementerio.

\begin{tabular}{|c|c|c|c|c|c|c|}
\hline VARIABLE & SEXO & EDAD & DEFORMACION & $\overline{\mathrm{GL}}$ & CEMENTERIO & GL \\
\hline DEFORMACION & & & & & $24,844^{*}$ & \\
\hline SEXO & & & 2,689 & & $9,000^{*}$ & \\
\hline EDAD & 2,959 & & 3,685 & & 8.136 & \\
\hline SURCOS DEL FRONTAL & 1,914 & 2,668 & 7,372 & & 5,087 & \\
\hline FORAMEN SUPRAORBITARIO & 0,166 & 2,992 & 9,137 & & 2,426 & \\
\hline ESCOTADURA SUPRAORBITARIA & 0,074 & 0,752 & 3,841 & & 0,241 & \\
\hline FORAMEN O ESCOTA. FRONTAL & 0.490 & 1,545 & 1,837 & & 0,157 & \\
\hline TUBERCULO TROCLLEAR & 0,037 & 3,316 & 1,936 & & 2,746 & \\
\hline FORAMEN ETMOI.ANTER.SUTURA. & 0,028 & 5,214 & 2,530 & & 2,283 & \\
\hline SUTURA INFRAORBITARIA & 0,345 & $\overline{0,594}$ & 1,869 & & 1.680 & \\
\hline FORAMEN INFRAORBIT.ACCES. & 0,580 & 0,030 & 0,492 & & 1,691 & \\
\hline FORAMEN CIGOMATICO FACIAL & 0,007 & 0,448 & $\overline{0,392}$ & & 0,498 & \\
\hline TUBRERC ULO CIGOMAXILAR & $7,025^{*}$ & 4,767 & 4,644 & & 9,660 & \\
\hline TUBEROSIDAD MALAR & $7,313^{* *}$ & 2,376 & 2,474 & & 3,102 & \\
\hline TUBERCULO MARGINAL & 0,433 & 1,786 & 4,290 & & 0,114 & \\
\hline HUESO PTERICO & 0,045 & 0,620 & 1,800 & & 3,535 & \\
\hline HUESO ESCAM.PARIET.TEMPORAL & 0,356 & 1,657 & 4,802 & & 5,761 & \\
\hline HUESO ASTERICO & 0,186 & 0,335 & 6,467 & & 1,499 & \\
\hline RAMA EMIS ARIA ART.MEN.MED. & 0,167 & 0,182 & 2,527 & & 1,243 & \\
\hline ART.TEMP.PROFUNDA POSTERIOR & 0,104 & 4,550 & 9,059 & & 6,163 & \\
\hline FORAMEN PALAT.MEN.ACCESORIO & 0,003 & 2,376 & 0,778 & & 0,605 & \\
\hline ESPOLONES ALA PTER.LATERAL & 2,514 & 0,608 & 0,209 & & 3,071 & \\
\hline FORAMEN EMISARIO ESFENOIDAL & 0,069 & 3,467 & 5,421 & & 4,829 & \\
\hline DEHISCENCIA LAM. TIMPANICA & 0,0 & 0,252 & 1,694 & & 2,834 & \\
\hline FOR. MARGIN. LAM TIMPÁNICA & 0,023 & 1,819 & 11,734 & & $5,29 !$ & \\
\hline TUBERCULO PRECONDILEO & 0,002 & 1,386 & 0,994 & & 1,728 & \\
\hline FORAMEN CONDILEO INTERMEDIO & 0,184 & 3,203 & 5,078 & & 6,818 & \\
\hline APOFISIS PARAMASTOIDE & 0,262 & 0,096 & 2,014 & & 0,212 & \\
\hline LIGAMENTO APICAL OSIFICADO & 0,768 & 0,200 & 1,063 & & 2.916 & \\
\hline FORAMEN HIPOOGLO.DOBLE & 0,952 & 0,118 & 3,599 & & 1,498 & \\
\hline CANAL CONDILEO POSTERIOR & 0,708 & 0,852 & 2,438 & & 6,135 & \\
\hline HUESO APICAL O LAMBDÁ TICO. & 2,919 & 4,624 & 0,232 & & 2,717 & \\
\hline HUESO SUTURA LAMBDICA & 0,351 & 3,702 & 0,158 & & 2,912 & \\
\hline HUESO EPACTAL & 0,020 & 0,135 & 2,024 & & 1,744 & \\
\hline PRESENCIA LINEA NUCAL ALTA & 0,004 & 1,175 & 0,802 & & 1,616 & \\
\hline WORMIANO OCCIPITO MASTOIDEO & 0,019 & 0,936 & 1,459 & & 4,996 & \\
\hline FORAMEN MASTOIDEO EXSUTU. & 1,863 & 1,566 & 8,115 & & 9,396 & \\
\hline APOFISIS MASTOIDES ESCOTADA & $0, \overline{134}$ & 0,475 & 0,173 & & 3,365 & \\
\hline FORAMEN PARIETAL & 0,068 & 0,697 & 1,006 & & 0,087 & \\
\hline
\end{tabular}

${ }^{*} \mathrm{y}^{* *}$ rechazos de las hipótes is de nulidad a los niveles del 0,05 y $0,0 \mid$ de probabilidad

de los rasgos considerados con la excepción del tubérculo cigomaxilar y la tuberosidad malar.

\section{Variación etaria}

En la tabla 2 se dan los valores de las pruebas de independencia referida a la distribución de los rasgos morfológicos gruesos en función de la edad de los cráneos. En este diseño se han agrupado las clases prerreproductivas $(1,2$ y 3$)$ por el escaso número de ejemplares disponibles. Los principales resultados indican que un conjunto de varia- bles morfoscópicas ocurren con independencia de la variación etaria, como por ejemplo el tipo de deformación, la conservación, la prominencia de la glabela, la forma de las órbitas, el vertex, el occipucio, etc., mientras que otras se muestran estrechamente asociadas como el tamaño del cráneo, los relieves óseos, la forma de la cara, el tamaño de los arcos superciliares, la abertura nasal y los huesos malares, cuyas categorías de menor desarrollo son más frecuentes en las clases de menor edad. El desgaste dentario sigue un patrón de edad creciente en forma neta, la sutura metópica 
Tabla 4:

Variables métricas, dócimas de hipótesis (Valores $F$ ) referidas a las diferencias debidas al sexo, la edad, la deformación y el cementerio

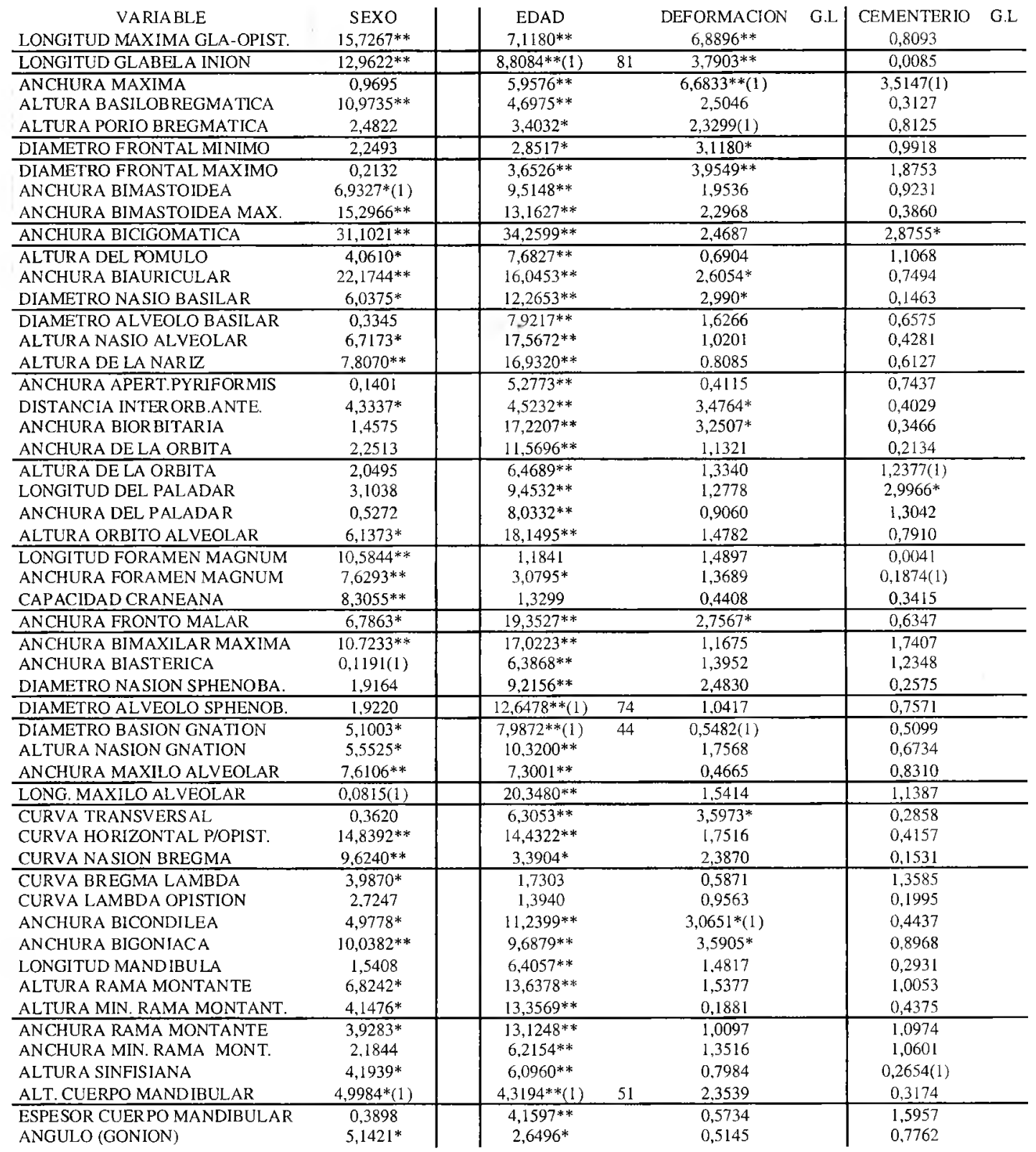

* y ** rechazo de la hipótesis de nulidad a los niveles del 0,05 y 0,01 de probabilidad, (1) heterogeneidad de varianzas al nivel 0,05 de probabilidad. 
desaparece, el frontal se inclina progresivamente, el surco retroglabelar se marca más, igual que la prominencia de los huesos nasales y el desarrollo de los arcos cigomáticos, entre otras características.

Los cambios antes señalados indicadores de un efecto dependiente de la edad de desarrollo de los individuos se acompañan de una evidente variación métrica tal como se pone de manifiesto en la Tabla 4, en la cual se consignan los resultados de las dócimas de hipótesis referidas a las diferencias entre los valores medios. Las pruebas previas referidas a la distribución de las variables indicaron algunos problemas de curtosis y dos casos de asimetrías al 0,01 de probabilidad (la anchura biastérica y la altura sinfisiana) en alguna clase de edad. También se comprobaron varianzas heterogéneas al nivel del 0,05 en la longitud antero posterior glabela inion, el diámetro alvéolo sphenobasion y la altura nasion gnation, y al nivel del 0,01 en la altura del cuerpo mandibular. El efecto de la edad se manifiesta en la mayoría de las características métricas estudiadas a dos niveles de significación. En cambio, carecemos de pruebas suficientes para comprobar la variación etaria en el caso de la capacidad craneana, la longitud del foramen, la curva bregma lambda y lambda opistion. En todos los casos se observa un claro patrón de crecimiento gradual.

Los atributos menores se manifestaron insensibles a la variación entre ejemplares adultos, maduros y seniles tal como puede apreciarse en la tabla 3 , en la cual figuran los resultados de los valores $\mathrm{Chi}^{2}$ correspondientes a las dócimas de independencia para estos caracteres. Este es un resultado de nuestro diseño que no incluyó individuos infantiles y juveniles. En el futuro, si se cuenta con esta información es posible que se pueda comprobar la influencia de la variación etaria en la distribución de estos rasgos.

\section{Deformación craneana artificial}

En las tablas 2, 3 y 4 se consignan los resultados de las dócimas de hipótesis referidas al análisis de las variaciones producidas por la deformación artificial en la morfogénesis del cráneo desde el punto de vista de características anatómicas gruesas, de las variables métricas y de los atributos meno- res respectivamente.

No hemos comprobado variaciones significativas producidas por la práctica cultural de diversos tipos (tabular erecta y oblicua, circular erecta y oblicua) en numerosos atributos mayores como la forma de la cara, la presencia de sutura metópica, el tamaño de los arcos superciliares, la forma de las órbitas, el tamaño de las aberturas nasales, el tamaño de los malares, etc. Pero la acción de los aparatos deformantes, como era de esperar, si parece ponerse de manifiesto a nivel de la glabela y del vertex, de la inclinación del frontal y de la altura de la bóveda, en la protuberancia occipital externa, del perfil craneano y en la presencia de plagiocefalía, etc. (Tabla 2). Tampoco se observó una asociación entre los distintos tipos deformatorios y el desgaste dentario.

Del total de variables métricas analizadas (Tabla 4), no podemos tomar en cuenta los resultados obtenidos con respecto a la anchura de la órbita, la anchura bigoníaca, la altura sinfisiana y el ángulo gonion, pues presentan distribuciones asimétricas, y la anchura máxima, la altura porio bregmática, el diámetro basion gnation y la anchura bicondílea que presentan varianzas heterogéneas al 0,05 de probabilidad. El factor deformación se puso en evidencia particularmente a nivel de la longitud y anchura del cráneo, de los diámetros frontales (tabla 4), anchura biauricular, diámetro nasio basilar, distancia interorbitaria, anchura biorbitaria, anchura fronto malar, etc. Existe un conjunto de mediciones que parecen ser invariantes, entre las que se destaca la capacidad craneana.

Con respecto a los atributos menores (Tabla 3) nada hace pensar en una distribución diferente entre los distintos tipos deformatorios y los ejemplares normales.

\section{Variación entre cementerios}

En las tablas 2, 3 y 4 se presentan los resultados de las dócima de hipótesis referidas a la distribución de los tres conjuntos de variables analizados en este trabajo en función de los cuatro cementerios.

Los datos disponibles no permiten comprobar una 
variación apreciable de la estructura por edades entre los distintos componentes, pero si en lo que respecta al sexo y a la deformación artificial (Tabla 2). Del conjunto de atributos gruesos analizados sólo nueve presentan proporciones diferentes entre cementerios. La distribución de los sexos varia con respecto a la esperada en el cementerio A. Aquí se produce una inversión en las proporciones normales, observándose un exceso de ejemplares femeninos y una escasa ocurrencia de masculinos. La deformación artificial también se encuentra asociada con el tipo de cementerio: los modelos circulares ocurren con mayor frecuencia y los tabulares oblicuos no existen en la unidad D, y en la designada con $\mathrm{H}$ predominan los tabulares oblicuos y están ausentes los circulares erectos. Estas comprobaciones sin dudas afectan las diferencias observadas con respecto a las características anatómicas gruesas, cuya distribución no puede ser analizada en forma independiente del sexo, de la edad o del tipo de deformación. Los relieves óseos muy marcados son más probables entre los individuos del componente $\mathbf{d}$ y muy escasos entre los de a; en aquel se presentan con mayor frecuencia las glabelas prominentes, las cuales se tornan raras en el $\mathbf{c}$. Los ejemplares $\mathbf{d}$ son en general de mayor tamaño que los del sitio a, en donde son más frecuentes las caras anchas y escasean los arcos superciliares marcados. Los frontales verticales predominan en a. En el sitio d, los cráneos son

Tabla 5:

Reconstrucción de la tabla de vida para la población de Pisagua considerando la muestra completa.

\begin{tabular}{|c|c|c|c|c|c|c|c|c|}
\hline $\begin{array}{c}\text { Clases de edad } \\
0-2\end{array}$ & $\begin{array}{c}\text { Rango } \\
\text { Int } \\
2\end{array}$ & 0 & $\mathrm{dx}$ & $1 \mathrm{x}$ & $\mathrm{qx}$ & 937,5 & 2445,45 & 24.45 \\
\hline $3-12$ & 10 & 15 & 12,5 & 87,5 & & 502,5 & 1507,95 & 17,23 \\
\hline $13-18$ & 6 & 9 & 7,5 & 80,0 & & 470,1 & 1005,45 & 12,57 \\
\hline $19-24$ & 6 & 4 & 3,3 & 76,7 & & 302,25 & 535,35 & 6,98 \\
\hline $25-29$ & 5 & 39 & 32,5 & 44,2 & & 182,7 & 233,1 & 5,27 \\
\hline $30-35$ & 6 & 33 & 27,5 & 16,7 & & 50,4 & 50,4 & 3,02 \\
\hline $\begin{array}{c}36- \\
\text { total }\end{array}$ & 120 & 20 & 16,6 & 0,1 & & 0,0 & 0,0 & 0,0 \\
\hline
\end{tabular}

Dx: número de muertos, dx: porcentaje de muertos; lx: sobrevivientes; qx: probabilidad de muerte; Lx: total de años vividos; Tx: total de años vividos después del nacimiento; $\mathrm{e}^{\circ}$ : esperanza de vida.

Tabla 6:

Diferencias entre cementerios. Valor $D_{3}^{2}$ entre pares de grupos con la correspondiente dócima de hipótesis $F$.

\begin{tabular}{ccc|c|c} 
& $\mathrm{D}$ & $\mathrm{C}$ & $\mathrm{A}$ & $\mathrm{H}$ \\
$\mathrm{D}$ & 0,000000 &, 516017 &, 395027 &, 701048 \\
$\mathrm{C}$ & 1,248634 & 0,000000 & 1,410033 & 2,082083 \\
\hline $\mathrm{A}$ & 1,148881 & $4,458101^{* *}$ & 0,000000 &, 708641 \\
\hline $\mathrm{H}$ & 1.744245 & $5.561569 * *$ & 2,323863 & 0,000000 \\
\hline
\end{tabular}

Distancias $\mathrm{D}_{3}^{2}$ he mimatriz superior; Valores $\mathrm{F}$ hemimatriz inferior g.I $=3$ y $70 ; * * \mathrm{p}<0,001$ 
altos y de perfil ovoide y en $\mathrm{C}$ hay más pentagonoides, etc. EI desgaste dentario se distribuye homogéneamente entre los distintos cementerios.

En cambio, la distribución de los rasgos no métricos permanece invariante (Tabla 3) pues no fue posible obtener pruebas fehacientes de variación entre componentes cronocontextuales. Con respecto a las variables métricas, se repite la observación anterior. Con la excepción de algunos rechazos de hipótesis vinculadas con distribuciones distintas de la normal y heterogeneidad de varianzas, las pruebas de diferencias entre medias no revelaron variación alguna entre cementerios (Tabla 4). Los cambios verificados en la anchura bicigomática y en la longitud del paladar son relativamente débiles (al 0,05 de probabilidad).

La igualdad entre los vectores medios de cada par de cementerios fue puesta a prueba en forma simultánea para un conjunto de 36 variables métricas, empleando datos libres de los efectos sexo, edad y deformación artificial. La aplicación de un procedimiento de selección de variables por su mayor poder discriminante permitió comprobar la existencia de variabilidad demostrable estadísticamente solamente para dos mediciones: la longitud del paladar y el diámetro alveolo sphenobasion, siguiéndoles en orden de importancia la anchura bimaxilar máxima. Con estas tres variables se calcularon los valores $D^{2}$ de la Tabla 6, y el gráfico de la Figura 3 fue dibujado con las dos primeras componentes discriminantes canónicas. Los resultados obtenidos indican claramente que las diferencias entre componentes son escasas desde el punto de vista estadístico. Se observa que los materiales del cementerio c son los que más se apartan del resto y que las elipses de equiprobabilidad al $90 \%$ no son similares para cada muestra indicando cambios en la varianza.

\section{Paleodemografía}

En la tabla 5 se presentan los resultados del análisis de la tabla de vida para el total de la muestra disponible de Pisagua los cuales deben ser tomados como valores medios para el intervalo cronológico representado por la muestra. La dis-

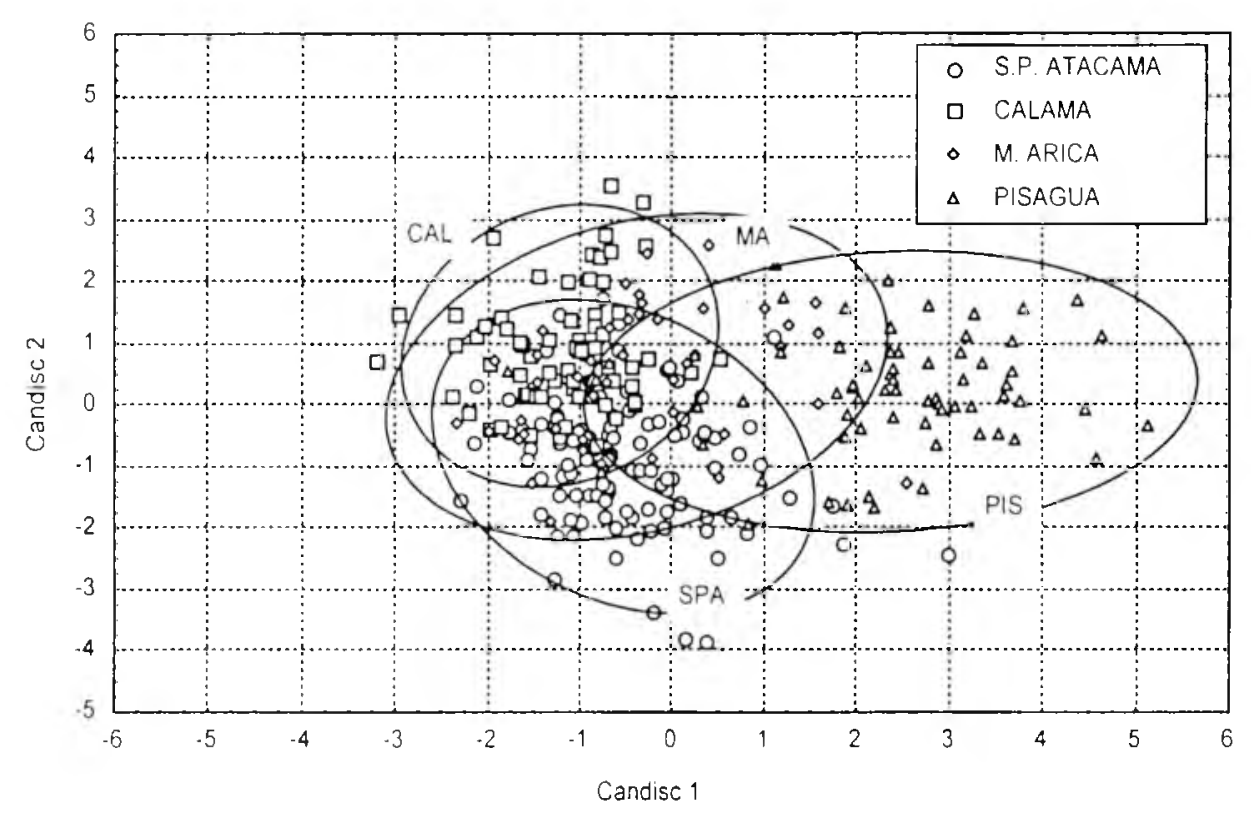

Figura 2:

Distribución de las observaciones de cada localidad en función de sus valores en la coordenada discriminante canónica 1 y 2; elipses de equiprobabilidad al 95\% 
tribución por edades parece consistente con la conocida para otras poblaciones prehistóricas (cfr. Bennett, 1973; Ubelaker, 1974; Quevedo, 1976; Benfer, 1984; Kamps, 1984). Se observa una mayor proporción en las categorías infantil, adulto y siguientes a ésta, en las cuales el riesgo de muerte era mayor por las condiciones de vida. Entre los distintos componentes parecería existir un desigual balance entre clases pre y postrreproductivas, pero lamentablemente con los datos disponibles no es posible demostrarlo por el momento. Considerando la muestra sin discriminar en fases cronocontextuales, en la Población de Pisagua la esperanza de vida al nacimiento era de 24 años. Una persona que llegaba a los 35 años podía esperar vivir tres años más. Entre los 13 y los 24 años la probabilidad de muerte era baja, incrementándose substancialmente con la edad. Los momentos de mayor riesgo eran cerca de los dos años y durante la edad adulta. Lamentablemente, no poseemos por el momento una estimación del período de acumulación de los restos, lo cual nos impide hacer un cálculo del número efectivo de la población.
A este nivel es posible realizar una síntesis para ensayar una caracterización, al estilo tradicional, de los cementerios de acuerdo con los materiales exhumados de cada uno de ellos (Figura 4, 5, 6 y 7):

El cementerio d se encuentra integrado por individuos de todas las edades y de ambos sexos en proporciones semejantes a las esperadas, que acostumbraban a deformarse la cabeza según los modelos circular erecto y oblicuo. Predominan los cráneos grandes con relieves óseos en general bien desarrollados, glabela prominente, bóveda mediana a alta, perfil ovoide y foramen magnun circular. Los materiales se encuentran bien conservados, algunos todavía con restos de tejidos adheridos. Existen casos de exostosis del conducto auditivo externo y un ejemplo de M3 incluído, además de la sutura esfenobasilar abierta. Hay una observación de proceso alveolar en PM2 superior derecho. Escasos rasgos de patologías. Una actualización de los datos de este cementerio fue realizada por Quevedo y Cocilovo (1995).

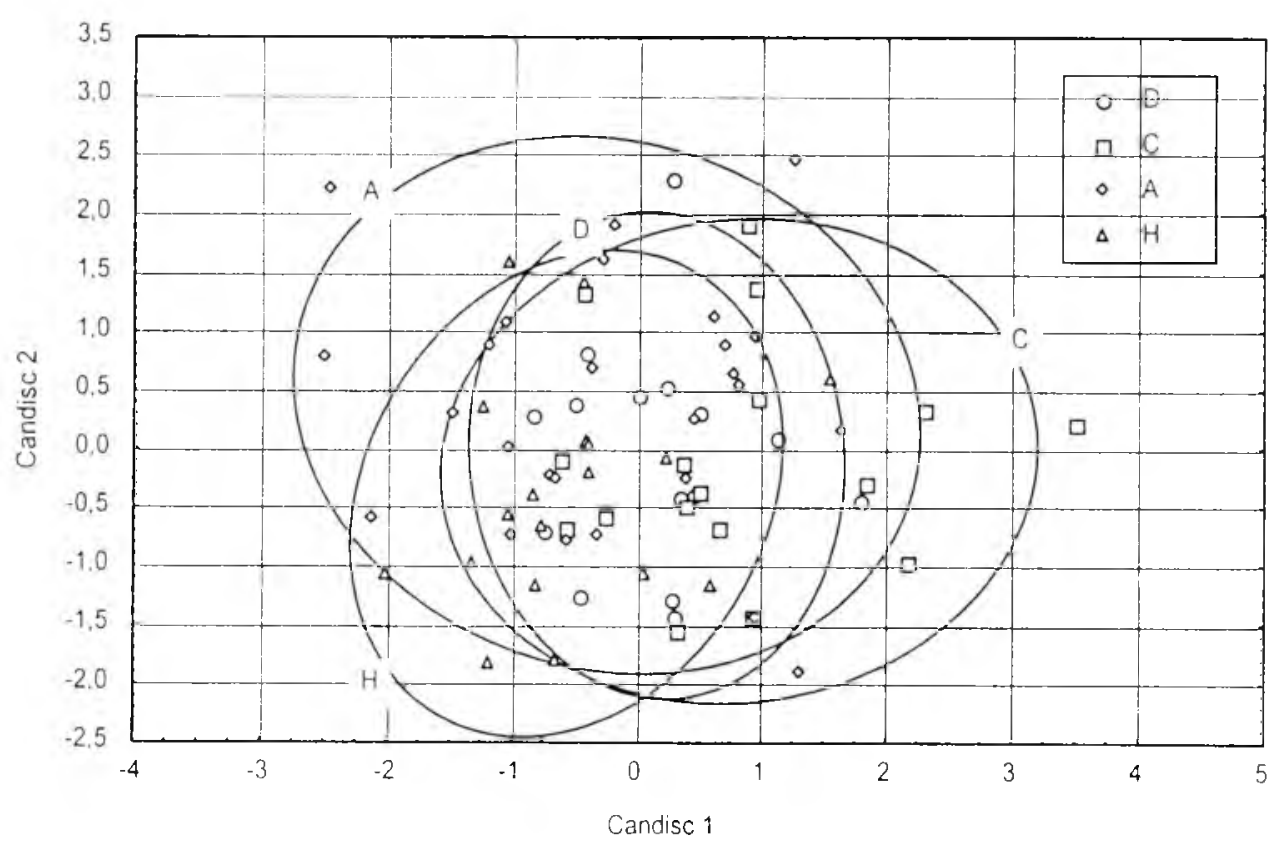

Figura 3:

Distribución de las observaciones por cementerio en función de las coordenadas discriminantes canónicas 1 y 2; elipses de equiprobabilidad al $90 \%$ 
El cementerio c posee también ejemplares de ambos sexos y diferentes edades en proporciones semejantes, pero predomina la costumbre de deformarse la cabeza de acuerdo con los tipos tabulares erectos y oblicuos. Disminuyen sensiblemente las glabelas prominentes y predomina el perfil craneano pentagonoide. Las restantes características no permiten obtener mayor información. Se observaron varios ejemplares que presentaban signos de osteoporosis generalizada con recuperación. Hay varios casos de conservación de tejidos desecados. Son frecuentes los procesos infecciosos con reabsorción alveolar, en particular podemos citar tres ejemplos a nivel de M1 izquierdo superior y M2 superior derecho. Hemos encontrado también un caso de M3 incluido y sinostosis esfenobasilar. Una revisión moderna de estos materiales fue realizada por Quevedo y Cocilovo (1996).

El cementerio a presenta una inversión en la proporción sexual estadísticamente significativa, predominando los ejemplares femeninos frente a los masculinos. Las clases de edad no difieren mayormente de la esperada para la población en conjunto. Se mantiene la práctica tabular erecta y oblicua. Son más probables los cráneos pequeños y muy escasos los grandes, al igual que los relieves óseos marcados. Predominan las caras angostas y los arcos superciliares muy desarrollados son infrecuentes. Los cráneos presentan frontales verticales, predominando los perfiles esfenoides. Hemos anotado casos de erupción del M3 y permanencia de la sutura esfeno basilar abierta. Hay frecuentes signos de osteoporosis y muchos casos de espongiohiperostosis con indicios de recuperación. También se observaron restos de tejidos adheridos a las piezas craneales. Son bastante frecuentes los procesos alveolares, particularmente en M1, M2 izquierdo superior y en el maxilar inferior con reabsorción alveolar en M3 y M2 derechos.

El cementerio $\mathbf{H}$ posee una proporción mayor de individuos masculinos que de femeninos, distribuidos en todas las clases de edad. Predominan las formas tabulares oblicuas y desaparecen las circulares erectas. El tamaño de los cráneos no varía y tampoco el aspecto de los relieves óseos, aunque son más frecuentes los arcos superciliares fuertes. Hay evidencias marcadas de osteoporósis generalizada en la bóveda y en el techo de las órbitas. Es también frecuente la reabsorción alveolar extendida, y los procesos alveolares en distintas

Tabla 7 :

Relaciones Biológicas. Muestras del Norte de Chile y cronologías estimadas para cada una de ellas

\begin{tabular}{|l|l|l|l|l|}
\hline Grupo & COLECCION & N & PERIODO & CRONOLOGIA \\
\hline 1 & & 17 & S.P.I & $800 \mathrm{aC}-100 \mathrm{dC}$ \\
\hline 2 & & 34 & S.P.II & $100-1000 \mathrm{dC}$ \\
\hline 3 & & 35 & S.P.III & $1000-1400 \mathrm{dC}$ \\
\hline 4 & & 28 & S.P.IV & $1400 \mathrm{y}+$ \\
\hline 5 & & 66 & TARDIO & $1450 \mathrm{dC}$ \\
\hline 6 & CALAMA (CHUNCHURI) & 44 & ARCAICO & $3000 \mathrm{aC}$ \\
\hline 7 & MORRO DE ARICA & 15 & AGR.TEMP & $0-300 \mathrm{dC}$ \\
\hline 8 & PISAGUA D & 17 & AGR.MEDIO & $300-1000 \mathrm{dC}$ \\
\hline 9 & PISAGUA C & 23 & AGR.TARDIO & $300-1000 \mathrm{dC}$ \\
\hline 10 & PISAGUA A & PISAGUA H & AGR.TARDIO & $1000-1450 \mathrm{dC}$ \\
\hline
\end{tabular}


piezas.

\section{Relaciones y afinidades biológicas}

Empleando un conjunto de 13 variables se comparó en forma global la información métrica disponible entre Pisagua, San Pedro de Atacama, Morro de Arica y Calama. Estos resultados, fueron obtenidos a partir de los datos libres de efectos debidos al sexo, la edad y la deformación artificial y se exponen en las tablas 7, 8 y 9 , y en la Figura 2. En la primera observamos las muestras empleadas y las cronologías aproximadas para cada una de ellas, en la segunda se consignan los valores $\mathrm{D}^{2}$ entre pares de localidades y las dócimas de hipótesis referidas a la igualdad entre vectores medios, y en la tercera tabla se presentan los re- sultados de la reclasificación de las observaciones por medio de las funciones discriminantes. En la Figura 2 se consigna la distribución de las observaciones de acuerdo con las dos primeras coordenadas discriminantes canónicas; cada muestra se encuentra circunscripta por una elipse de equiprobabilidad al nivel del $95 \%$.

Las mediciones resultantes del procedimiento de selección en orden de importancia fueron: curva transversal, diámetro frontal máximo, altura basilo bregmática, anchura máxima, anchura del paladar, diámetro frontal mínimo, longitud del paladar, anchura de la nariz, anchura maxilo alveolar, anchura bicigomática, curva nasion bregma, altura orbitaria y diámetro nasion sphenobasion. Los dos primeros vectores propios del análisis discri-

Tabla 8:

Diferencias entre localidades. Valores $D^{2}$ entre pares de grupos con la correspondiente dócima de hipótesis $F$

\begin{tabular}{|l|c|c|c|c|}
\hline & S. P. ATACAMA & CALAMA & M. ARICA & PISAGUA \\
\hline S. P ATACAMA & 0,000000 & 3,88334 & 3,173198 & 9,54857 \\
\hline CALAMA & 11,81094 & 0,00000 & 3,430737 & 13,56392 \\
\hline M.ARICA & 7,28496 & 6,54414 & 0,000000 & 9,23389 \\
\hline PISAGUA & 29,32347 & 32,74001 & 17,72059 & 0,00000 \\
\hline
\end{tabular}

Valores $D_{13}^{2}$ hemimatriz superior y Valores $F(p<0,0001$, g.l $=13$ y 275$)$ hemimatriz inferior

Tabla 9:

Matriz de clasificación, Filas: clasificaciones observadas, Columnas: clasificaciones estimadas

\begin{tabular}{|l|c|c|c|c|c|}
\hline & Porcentaje & S. P ATACAMA & CALAMA & M. ARICA & \\
\hline & Correcto & $\mathrm{p}=, 39175$ & $\mathrm{p}=, 22680$ & $\mathrm{p}=, 15 \mathrm{I} 20$ & $\mathrm{p}=, 23024$ \\
\hline S. P ATACAMA & 78,07018 & 89 & 12 & 9 & 4 \\
\hline CALAMA & 71,21212 & 15 & 47 & 4 & 0 \\
\hline M. ARICA & 43,18182 & 14 & 6 & 19 & 5 \\
\hline PISAGUA & 85,07462 & 6 & 3 & 1 & 57 \\
\hline Total & 72,85223 & 124 & 68 & 33 & 66 \\
\hline
\end{tabular}


minante canónico explican el $90 \%$ de la variabilidad total. El valor lambda de Wilk fue 0,1897, $\mathrm{F}=15$ y $737 \mathrm{~g} . \mathrm{l} .(\mathrm{p}<0,000)$ indicando diferencias globales significativas entre los vectores medios de las distintas localidades. Las variables que más contribuyeron en la ponderación del primer vector propio fueron: curva transversal, diámetro frontal máximo, altura basilo bregmática y anchura máxima, y en el segundo vector propio: longitud del paladar, anchura máxima, anchura del paladar y curva nasion bregma. Las distancias $\mathrm{D}^{2} \mathrm{de}$ Mahalanobis entre pares localidades indicaron también la existencias de diferencias significativas, siendo Pisagua la muestra que más se distingue del resto.

La proporción de observaciones clasificadas dentro del propio grupo es variable, siendo el valor más alto el correspondiente a Pisagua y el más bajo, a Morro de Arica. La composición generalizada de esta última muestra se pone en evidencia por la mayor cantidad de observaciones propias asignadas a otro grupo entre los que se destaca San Pedro de Atacama.

\section{Discusión y conclusiones}

El análisis y la discusión de los resultados obtenidos en el conjunto de experiencias aquí relatadas deben ser realizados de acuerdo con los supuestos que condicionan las dócimas de hipótesis y con la naturaleza de los diseños experimentales aplicados. El número total de observaciones es todavía escaso para sustentar la información derivada en forma más sólida, pero a la vez, esta constituye la única fuente de conocimientos disponibles por el momento. En los procesos estadísticos de atributos debemos tener en cuenta la posible correlación entre factores como la edad, el sexo, la deformación y el tipo de cementerio, y entre estos y las características anatómicas empleadas. Por ejemplo, mal podemos evaluar la variación de una distribución asociada con el tipo de componente contextual si ésta es producida en forma subyacente por la influencia del dimorfismo sexual. Esto fue probado en cada oportunidad antes de las dócimas realizadas para rasgos morfológicos gruesos y no métricos. Con respecto a las variables continuas, el problema es similar, sólo que con éstas intentamos una forma de superarlo analizando por separado cada factor habiendo eliminado previamente el efecto de los restantes. Los supuestos de distribución normal y de homocedasticidad fueron evaluados para cada variable, lo cual fortalece las dócimas y las inferencias realizadas en las estimación de las diferencias entre valores medios. Hemos tomado con prudencia estos resultados cuando dichos supuestos no se cumplían al 0,05 de probabilidad y no eran sistemáticos, pero sí hemos desechado del análisis una característica cuando presentaba problemas al nivel 0,01. De todas formas estos resultados están expuestos y el lector puede tomar una decisión por sí mismo. A continuación, comentamos los principales temas de acuerdo como fueron expuestos anteriormente, intentando destacar los aspectos más importantes en una apretada síntesis.

El dimorfismo sexual puede ser apreciado por la morfología externa de los cráneos, empleando un conjunto estandarizado de características anatómicas gruesas, con independencia de las variaciones producidas por la edad y la deformación artificial. Se comprueba claramente la falta de pruebas para demostrar la asociación entre el sexo, la edad y la deformación artificial, aunque el primero y el último factor sí están vinculados con el tipo de cementerio pero no entre si. Esto parecería determinar una situación de incertidumbre en el momento de evaluar objetivamente las relaciones de causalidad entre un determinado carácter y un efecto dado, pero teniendo en cuenta la naturaleza del rasgo anatómico considerado, podemos avanzar en consideraciones más confiables. En la tabla 2 se observan los rasgos que más se asocian con la expresión del sexo en el cráneo y que por ello constituyen buenos indicadores para el diagnóstico. Esto es algo esperado de acuerdo con la bibliografía sobre el tema (Borovansky, 1936; Bass, 1971; Genovés, 1962; Boyd y Trevor, 1953; Larnach y Freedman, 1964; Keen, 1950; Ferembach, Schwidetzky y Stloukal, 1979). Existe también un conjunto de características que permanecen invariantes, entre las cuales el desgaste dentario podría indicar una dieta de naturaleza similar entre uno y otro sexo.

La distribución independiente de la edad con respecto a los restantes factores analizados, en principio, puede asegurar la evaluación de sus efectos directos sobre los rasgos anatómicos gruesos. El conjunto asociado con la variación etaria son fun- 
damentalmente aquellos que reflejan cambios de tamaño en determinadas porciones óseas. Desde el punto de la bibliografía existente sobre el tema esto no constituye ninguna novedad (Bass, 1971; Genovés, 1962; Boyd y Trevor, 1953; Ferembach, Schwidetzky y Stloukal, 1979) excepto por la forma de probarlo. El mayor o menor desarrollo de un accidente anatómico también se encuentra relacionado con la expresión del dimorfismo sexual, sin embargo, existen dos rasgos el desgaste dentario y los arcos cigomáticos (desde criptocigia a fenocigia) que parecen seguir un patrón de desarrollo de acuerdo con la edad y sin relación con el dimorfismo sexual. Por esta razón pueden ponderarse más que los restantes en trabajos futuros de determinación de las distintas fases de edad.

El efecto de la deformación artificial también es evidente a partir del examen de características anatómicas gruesas en aquellas porciones en donde es de esperar que se alteren por la práctica cultural como la prominencia de la glabela, del vertex y la mayor o menor inclinación del frontal, la altura de la bóveda, el aspecto de la escama occipital, el perfil craneano, la existencia de plagiocefalía y los arcos cigomáticos (criptocigia y fenocigia). Aunque no tenemos pruebas de la aplicación de tipos deformatorios diferentes para uno u otro sexo, algunos rasgos indicadores, también se distribuyen diferencialmente de acuerdo con el sexo lo que podría confundir la evaluación de los verdaderos efectos producidos por la deformación artificial. Sin embargo, un resultado interesante es la asociación con el tamaño y el peso del cráneo: en la deformación tabular oblicua predominan los ejemplares más livianos y pequeños, encontrándose los ejemplares más grandes entre los deformados circulares oblicuos. Las glabelas prominentes predominan exclusivamente entre los circulares igual que el vertex pronunciado y el frontal muy inclinado. Las bóvedas altas son características de los circulares erectos, en cambio las bajas son típicas de los tabulares oblicuos. Las protuberancias occipitales externas son de desarrollo mediano a fuerte, el perfil ovoide y la criptocigia predominan entre los circulares, mientras que la plagiocefalía se asocia con las formas tabulares. La discusión de estas comprobaciones con lo tradicionalmente propuesto (Dembo e Imbelloni, 1938) es difícil por la sencillez de las descripciones disponibles, aún en estudios reali- zados con anterioridad en los mismos materiales (Munizaga, 1964a y 1974). Un hecho que debemos anotar es la homogeneidad del desgaste dentario entre los ejemplares con distintos tipos deformatorios e incluso entre aquellos sin deformación.

Las diferencias morfológicas halladas entre cementerios son más difíciles de evaluar pues el sexo y la deformación artificial se distribuyen fuertemente asociados con el tipo de componente cronocontextual. Por ejemplo, mientras que en la unidad " $D$ " predominan los individuos de mayor tamaño y de relieves óseos marcados, en " $\mathrm{A}$ " son más frecuentes los cráneos pequeños y es escasa la categoría de ese atributo, precisamente en donde ocurre un mayor número de femeninos, predominan las caras angostas y escasean los arcos superciliares grandes, ambos rasgos característicos de este sexo. Las glabelas prominentes son más frecuentes en la unidad $\mathrm{D}$, en la cual también se da una mayor proporción de deformados circulares, práctica cultural que como hemos demostrado se asocia con un mayor desarrollo de esta porción anatómica. Algo similar ocurre con los restantes rasgos como la inclinación del frontal relacionado con el sexo y el tipo de deformación artificial, la altura de la bóveda y el perfil craneano. Finalmente nos queda una sola característica que es la forma del foramen magnun (relevada como circular o poligonal) no asociada con ningún otro factor y que aparece predominando en la primera categoría en el cementerio D. Lamentablemente, constituye una prueba muy débil para sustentar la diferenciación entre unidades cronocontextuales. Estos resultados hablan por si mismos sobre el escaso valor de las tradicionales descripciones morfológicas realizadas para postular tipos humanos o razas, sin dudas desarrolladas y aplicadas sin un adecuado control de la variabilidad debida al sexo, a la edad y a la deformación artificial. Otro hecho interesante es que el desgaste dentario se reparte en forma semejante entre los materiales de los distintos cementerios indicando poco cambio de la dieta.

La distribución de los atributos menores (rasgos discretos o no métricos) no ofrecen pruebas suficientes para demostrar su asociación con alguno de los factores de variación estudiados. De 36 características, sólo dos, el tubérculo cigomaxilar y 
la tuberosidad malar se encuentran vinculados con el sexo, el resto permanece realmente invariante con respecto a este factor, a la deformación artificial y a la variación etaria. Estos resultados no son compatibles con los obtenidos por otros autores que se han ocupado del tema, pero debemos advertir que nuestra muestra (con ejemplares adultos, maduros y seniles) no es adecuada para demostrar una asociación real con la edad pues no incluyó piezas infantiles y juveniles. Su invariancia con respecto a los citados factores es declarada por Sullivan (1922), Berry y Berry (1967), Perizonius (1979), Finnegan y Marcsik (1979), Ossemberg (1987) y Vives y Bertranpetit (1986). La influencia de la deformación artificial demostrada por Ossemberg (1970) no es muy sólida. Por otra parte, las comprobaciones de Pucciarelli (1972 y 1973) en materiales del N.O. argentino y de Patagonia no son confirmadas por nuestro trabajo. En Morro de Arica (Chile) no se pudo demostrar en forma indiscutible una relación de causalidad con el sexo, la edad y la deformación artificial (Martino et al., 1991). Buikstra (1976) tampoco encuentra pruebas concluyentes acerca de la influencia de la deformación artificial, pero sí la distribución de estas variables parece ser dependiente del desarrollo ontogenético. Las discrepancias observadas entre los distintas experiencias citadas, sin dudas se deben a la naturaleza de los datos y a los diseños experimentales aplicados en cada caso; por ejemplo, en muchos trabajos no puede asegurarse la independencia entre los propios factores ensayados, lo cual puede confundir los resultados presentados. Algo similar ocurre con el pionero trabajo de Munizaga (1964b) en poblaciones del Norte de Chile.

Tampoco pudimos encontrar distribuciones distintas a las esperadas de acuerdo al tipo de unidad funeraria. En base a esto último, la consecuencia es inmediata: con la muestra disponible no tenemos pruebas para suponer en Pisagua la existencia de más de un componente asociado con alguna unidad funeraria y diferente al de la población ancestral.

Desde el punto de vista métrico, la variabilidad es mayor y pudo ser demostrada en forma más prolija para cada efecto en particular. De 52 mediciones, 32 mostraron alguna variación con relación al dimorfismo sexual y 20 permanecieron inva- riantes. Las diferencias radican en la adquisición de dimensiones mayores en los varones con respecto a las mujeres, en el volumen endocraneano, en el desarrollo de la porción fronto-parietal del perfil sagital, en la anchura de la base y en el foramen magnun, en las dimensiones generales de la cara, y del maxilar inferior en cuanto a la anchura y al tamaño de las ramas ascendentes. Tanto uno como otro sexo adquieren un desarrollo igual a nivel de la anchura de la bóveda, de la altura porio bregmática, del crecimiento en anchura del frontal, de la longitud de la base craneal y de la pirámide facial, en las dimensiones de las órbitas y del paladar, etc. Estas comprobaciones, con las razonables diferencias producidas por otros diseños experimentales, coinciden con los resultados obtenidos con materiales de Patagonia, Perú y Norte de Chile (Cocilovo, 1973, 1975, 1978; Cocilovo et al., 1982, etc.), y con las observaciones de Keen (1950).

La edad constituye un importante factor de variación métrica pues casi la totalidad de las variables analizadas acusan diferencias marcadas entre las distintas clases etarias. El comportamiento de algunas de ellas siguen patrones diferentes, pero consistentes con lo esperado, a pesar de la escasa literatura existente sobre el tema. La muestra disponible y el especial algoritmo empleado, permitieron estudiar un fenómeno al cual pocas veces se tuvo acceso con materiales de poblaciones prehistóricas. También el análisis de los resultados obtenidos indica que algunas variables siguen un ritmo de crecimiento distinto, como por ejemplo la longitud del foramen magnun, la capacidad craneana y los segmentos parietal y occipital del perfil craneano sagital, las cuales alcanzan el valor adulto a una edad más temprana que el resto. La amplitud de las clases de edad existentes en esta colección no nos permitió, en estas medidas demostrar cambios estadísticamente significativos. Estas observaciones coinciden con las variaciones morfológicas declaradas por la literatura sobre el tema (Cocilovo, 1995).

En el análisis métrico de la deformación artificial, se han comparado los tipos tabular erecto, tabular oblicuo, circular erecto y circular oblicuo, con un grupo de ejemplares sin deformar, en forma simultánea. Por esta razón los resultados son globales y poco demostrativos del comportamiento 
de cada práctica en particular. En este punto, nuestro objetivo no fue brindar una caracterización métrica exacta de cada una de ellas, sino lograr una evaluación general del efecto sobre la morfología craneana. Se observa una influencia más acentuada sobre el neurocráneo y una extensión más ligera de la misma al esplacnocráneo. Los cambios comprobados son los esperados de acuerdo con trabajos anteriores (Cocilovo, 1973, 1975, 1978; Cocilovo et al., 1982).

Las diferencias métricas halladas entre los materiales de los distintos cementerios, una vez eliminados los efectos de la edad, el sexo y la deformación artificial, son sumamente escasas. Los materiales de la colección de Pisagua pueden considerarse como pertenecientes a una misma población, pues no obtuvimos pruebas suficientes para demostrar diferencias estadísticamente significativas entre las distintas unidades funerarias. Esta es una conclusión razonable, tanto a nivel de la morfoscopía gruesa, como de los rasgos no métri$\cos$ y de las variables métricas, aunque no podemos descartar que desde el punto de vista estadístico, un incremento futuro en los tamaños muestrales podría arrojar resultados distintos. Sin embargo consideramos que la prueba aquí presentada es muy sólida.

Por otra parte, no podemos ocultar que el conjunto de comprobaciones realizadas producen muchas dudas sobre las tradicionales clasificaciones raciales realizadas en América, tomando en cuenta características morfológicas gruesas, con una apoyatura nominal solamente de una métrica mal evaluada (cfr: Imbelloni, 1938, 1958; Bórmida, 195354; Munizaga, 1974, 1976). Los resultados de nuestro trabajo apoyan el empleo de atributos gruesos para la determinación del sexo, de la edad y de la deformación artificial, pero no para evaluar las diferencias entre cementerios cuando dichos factores no se distribuyen aleatoriamente $y$, aunque así lo fuera, este tipo de rasgos se encuentran muy vinculados con el grado de desarrollo de los individuos, siendo difícil evaluar con ellos la interacción entre los distintos efectos.

La corrección de los datos métricos originales, extrayendo el efecto del sexo, de la edad y de la deformación artificial, puede haber producido la eliminación de una gran proporción de variabili- dad ambiental. Si esto fue así, las pruebas realizadas adquieren particular significación para evaluar las reales diferencias entre los distintos cementerios aunque desde el punto de vista univariado ni multivariado no se haya logrado confirmar la estructuración de la población de Pisagua en cuatro componentes cronocontextuales. La variación residual restante puede ser, en un futuro, importante para comprender los mecanismos microevolutivos locales que operaron en el pasado.

Considerando los materiales de Pisagua como una unidad biológica local, estrechamente adaptada a la explotación de recursos marinos, complementados con productos continentales, durante un lapso entre 1400 a 1700 años, la evaluación de su tabla de vida en forma global proporciona una importante información sobre el proceso adaptativo local. La esperanza de vida de 24 años es mayor que la obtenida por Kamps (1984) para Camarones 14 (6000 AC), aunque se menciona un rango de $e_{x o}$ entre 20,7 y 24 años asociada con una tasa bruta de mortalidad de 45,1 o/oo. Para Pisagua este último valor llega a 41 o/oo. Un revisión más moderna con mayor información permitió realizar para el cementerio $C$ una estimación de $\mathrm{e}_{\mathrm{xo}}$ de 25,43 años con una tasa bruta de natimortalidad del 39 o/oo (Quevedo y Cocilovo, 1995). En materiales de Quitor 6 correspondientes a la fase tardía del desarrollo cultural de San Pedro de Atacama ( 900 a 1200 DC) se calculó una esperanza de vida al nacimiento de 26,61 años (Costa Junqueira, 1985). En el N.O. Argentino en el grupo de Las Pirguas, sometido a un alto impacto ambiental, correspondiente a una etapa temprana y media de la cultura Candelaria $(600 \mathrm{DC})$, se encontró para este parámetro un valor de 22,06 años (Baffi, 1989), mientras que en la Quebrada de Humahuaca, Cocilovo y Bordach (1985) en materiales tardíos del Pucará de Tilcara estimaron una cifra de 24,69 años. Estos distintos valores, sin duda se acusan diferencias en el modo de vida y en la estrategia reproductiva de las poblaciones cuya discusión exige la disponibilidad de mayor información sobre salud y nutrición, sobre el aprovechamiento de recursos y la magnitud de las interacciones biosociales.

La cuestión del origen, del desarrollo y de las relaciones biológicas de la población de Pisagua con 
el resto de los grupos del Norte de Chile es un problema complejo. La variación morfológica entre localidades, evaluada sin la influencia de los factores intrapoblacionales, es manifiesta desde el punto de vista estadístico. En efecto, Pisagua, Morro de Arica, San Pedro de Atacama y Calama, considerados globalmente, constituyen núcleos poblacionales diferentes. Las dos primeras series parecen pertenecer a entidades biológicas muy distintas, dicho esto a partir de pruebas con variables métricas y no métricas (Martino y Cocilovo, 1988). La observación de la Figura 2 es particularmente demostrativa con respecto a la composición de Pisagua pues la distribución de las observaciones en las dos primeras variables canónicas muestra una área de superposición con las otras localidades y a la derecha del gráfico otra área extensa particular que se proyecta en forma independiente en la dirección positiva de la primera componente discriminante. Esto indica la existencia en Pisagua de una cierta cantidad de variación vinculada con los restantes grupos y una proporción de variación nueva y diferente del resto, cuyo origen plantea un importante problema de investigación. Con estos resultados se podría postular en el Norte de Chile, la existencia de dos modelos fenotípicos: uno continental y otro litoral, pero hace falta el desarrollo de una investigación particular.

La historia biológica de los grupos arcaicos, $\sin$ dudas, es la clave para interpretar el desarrollo posterior de la población del norte de Chile. Las similitudes entre las muestras indican su origen a partir de un fondo genético común, y las diferencias, como en el caso particular de las novedades halladas en Pisagua por la existencia de procesos independientes en cada localidad. Por esta razón, en esta población es necesario pensar que el origen de la nueva variación puede haber sido local a partir de un fuerte proceso de diferenciación por aislamiento o extrarregional (migración). En este último caso, sería interesante probar la posible estructuración de la población arcaica a partir de la cual se originan los grupos formativos en dos o más sistemas biológicos dentro de los cuales la interacción fue mayor que entre ellos. Grupos arcaicos semejantes a Morro de Arica podrían haber sido, en parte, los responsables del poblamiento temprano del Valle de Azapa, de las cuencas in. termedias y de la zona del oasis de San Pedro de
Atacama. En el área de Pisagua, a pesar de que se registra la presencia de estos grupos desde el punto de vista arqueológico, lamentablemente los datos antropológicos son insuficientes para inferir sus características biológicas, aunque en la Figura 2 se distingue claramente una amplia área de superposición en las elipses de equiprobabilidad de Morro de Arica y de Pisagua. Durante el Formativo, parece ser más clara la diferenciación de estas dos entidades que, sin dudas, poseen raíces más antiguas en la región. Grupos semejantes a los que dejaron sus restos en el cementerio " $D$ ", de Pisagua habrían dado origen al desarrollo del segmento histórico de la población presentado en este trabajo, los cuales posiblemente se encuentren asociados con los del período agroalfarero más temprano de San Pedro de Atacama. Estas diferencias se asentuaron con el correr del tiempo, confiriendo a cada localidad, durante la influencia de Tiwanaku y en el período de Desarrollos Regionales, una personalidad propia que parece mantenerse durante la expansión incaica. Una explicación más detallada sobre los procesos microevolutivos ocurridos en San Pedro de Atacama y en la presente localidad se expone en los trabajos de Cocilovo (1995) y Varela (1997).

El poblamiento del Norte de Chile sin dudas es el resultado de procesos microevolutivos generados en áreas próximas que por milenios afectaron tanto el litoral norte como la zona de los oasis y cuencas intermedias. A las migraciones de amplio rango (desde fuera de la región: altiplano, selvas y N.O. argentino), propuestas y probadas por otros trabajos (Rothhammer et al., 1983; Rivera y Rothhammer, 1986), debemos sumar además, la influencia de los fenómenos de adaptación local y la interacción entre localidades. Las diferencias halladas pueden ser explicadas mediante la aplicación de un modelo basado en la migración, la selección y la deriva como factores fundamentales que producen cambios genéticos, para lo cual se torna necesario evaluar su participación individual y conjunta a través del aporte de variación nueva, del impacto ambiental y social sobre la misma, y del costo del proceso de adaptación que implicó la colonización de nuevos ambientes. Resulta imprescindible el aporte de información sobre la calidad de vida en determinados períodos y entre localidades para inferir su influencia juntamente con el flujo genético en la tasa de 

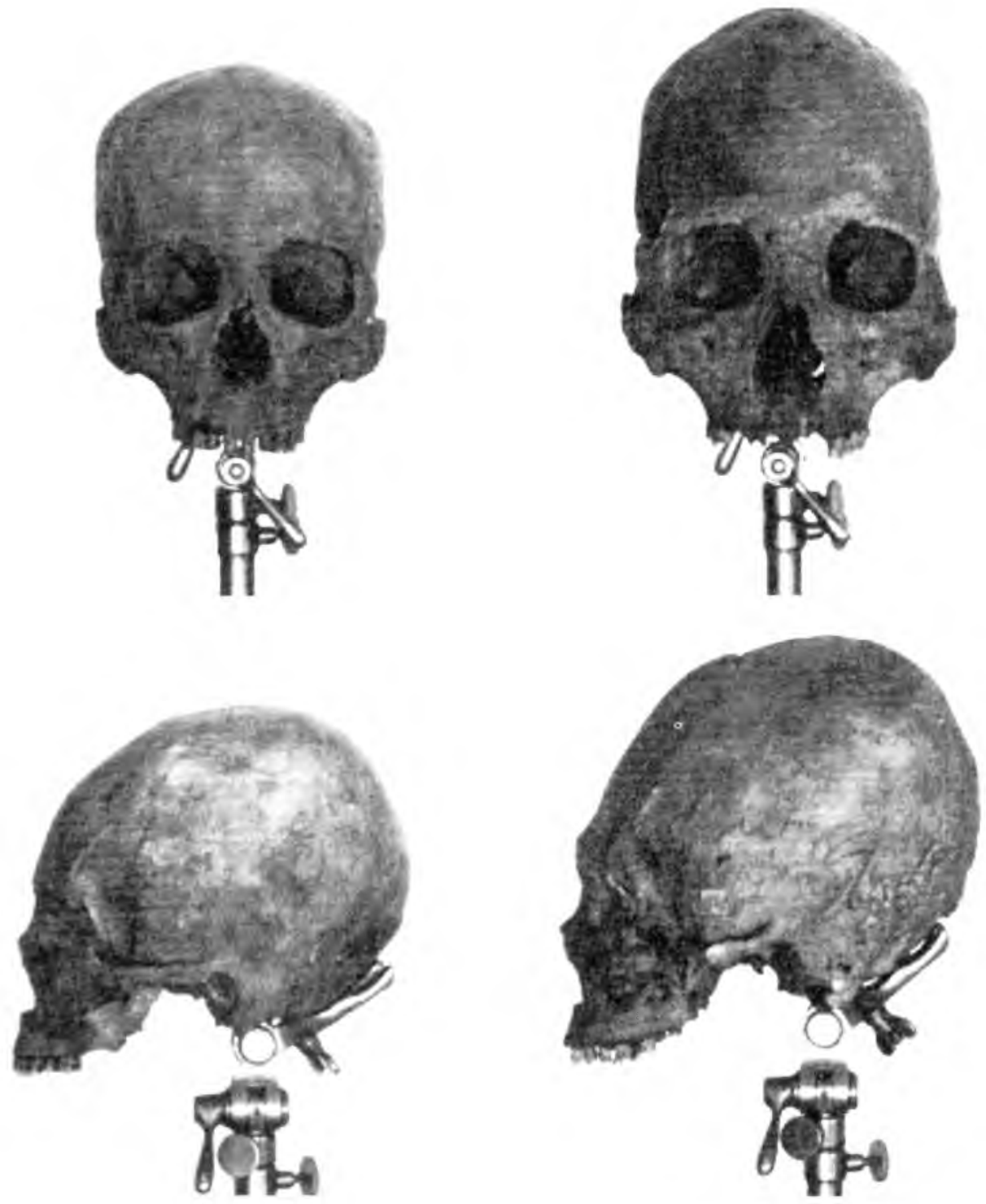

Figura 4:

CEMENTERIO D. Ejemplar 220, sin deformación, femenino, adulto maduro avanzado y Ejemplar 346, circular erecto, masculino, adulto maduro avanzado. 

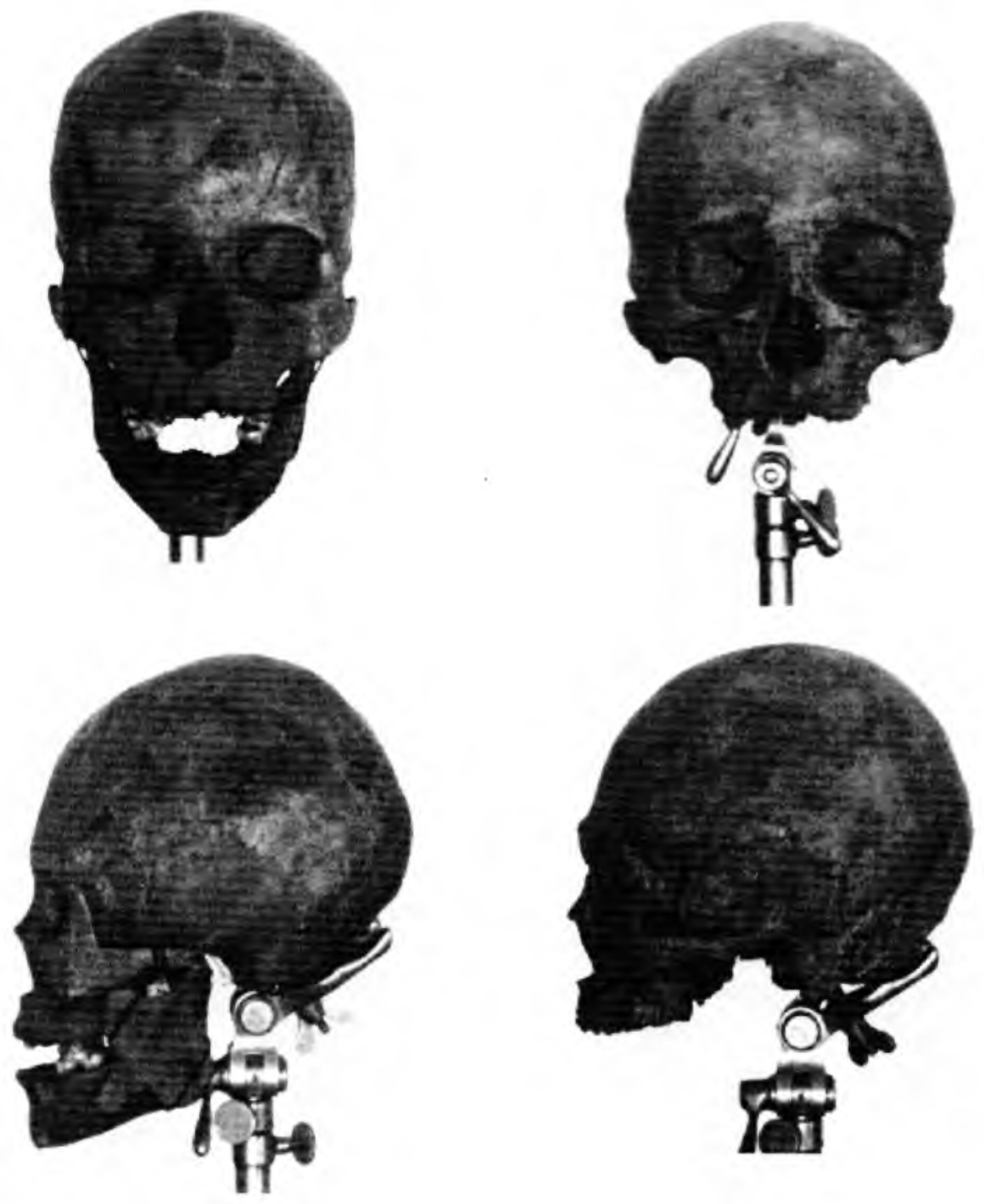

Figura 5:

Cementerio C, ejemplar 255, sin deformación, femenino adulto y ejemplar 259 , sin deformación, masculino, adulto maduro avanzado. 

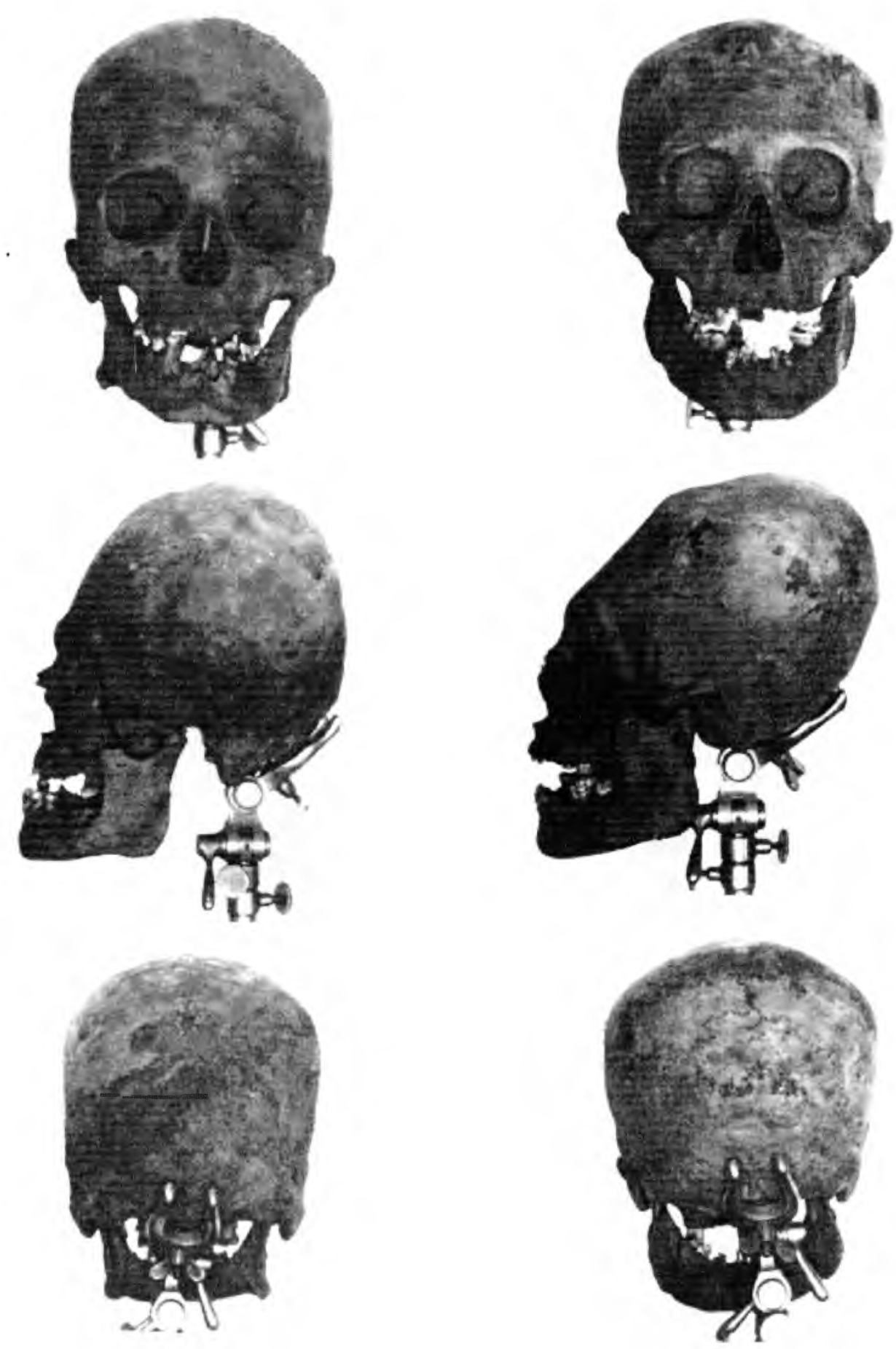

Figura 6:

Cementerio A. Ejemplar 337, tabular erecto, masculino, adulto maduro y Cementerio H, ejemplar 299, tabular oblicuo, masculino, adulto maduro. 

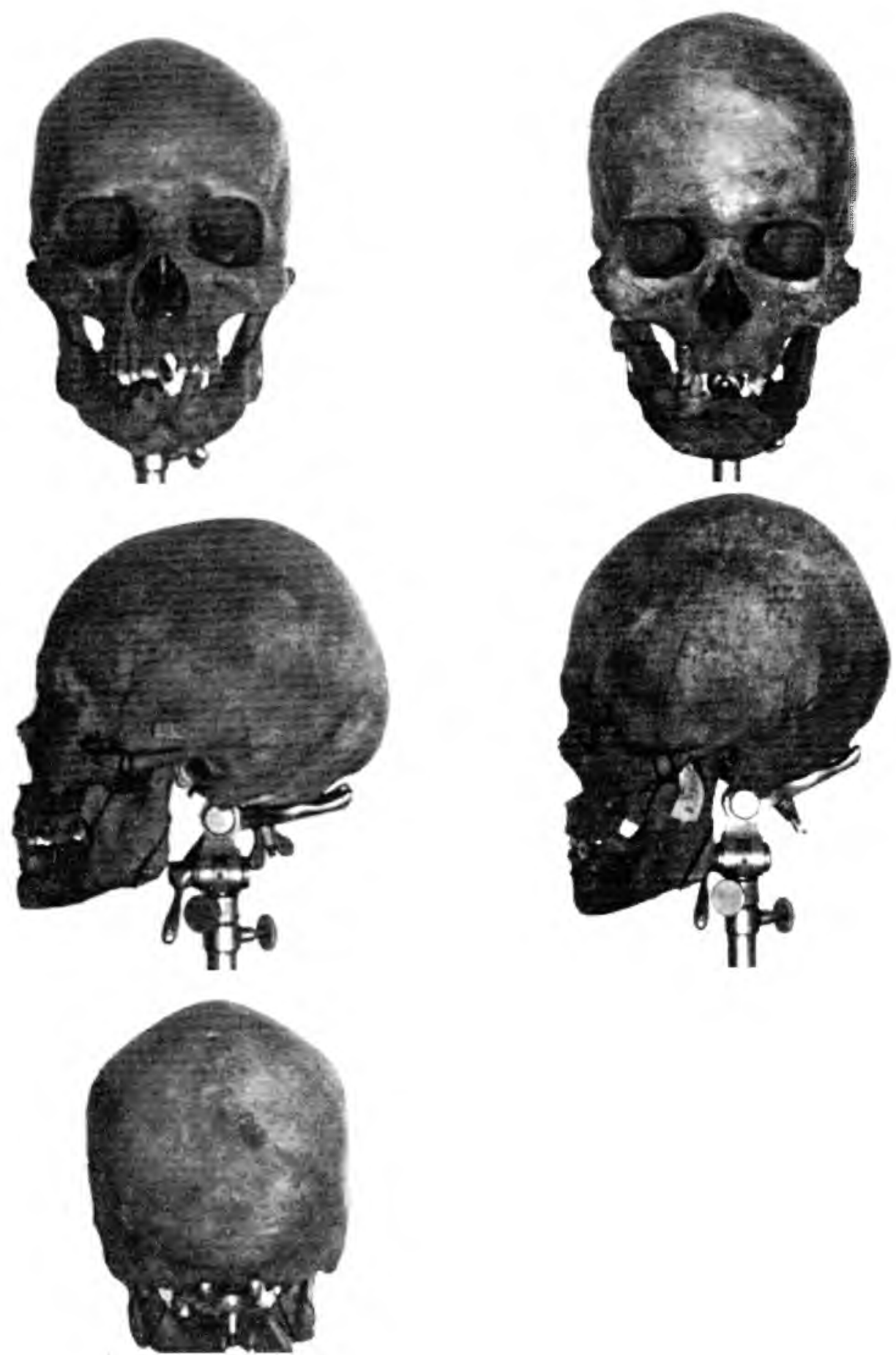

Figura 7:

Cementerio A, ejemplar 325, tabular erecto, femenino, adulto maduro y Cementerio C, ejemplar 264, tabular erecto, masculino, adulto maduro avanzado. 
microevolución por la ganancia o pérdida de variabilidad. Por ejemplo, la velocidad de cambio morfológico entre Morro de Arica y Pisagua, es mucho mayor que entre ella y San Pedro de Atacama. Esto está claramente indicando historias biológicas diferentes. Un conocimiento más adecuado de la estructura demográfica de cada grupo nos aportaría la información básica necesaria para inferir una de las posibles causas del proceso de microdiferenciación, así como la disponibilidad de un mayor número de muestras a nivel regional nos ayudará a comprender mejor la estructura genética de la población y la naturaleza de las interacciones biosociales.

\section{Agradecimientos}

En especial deseamos brindar un reconocido homenaje a la Dra. Grete Mostny, al Dr. Hans Niemeyer y al Dr. Francisco Rothhammer por el apoyo y el permanente estímulo que nos brindaron. Dejamos expresa constancia de nuestro sincero agradecimiento al personal de Museo Nacional de Historia Natural por su cooperación durante la preparación de la colección para la realización de los trabajos. También deseamos hacer extensivo nuestro agradecimiento a la Sra. Loreto Solé por su ayuda en el relevamiento de los datos y al Lic. Julio Sanhueza por habernos proporcionado información personal sobre la zona de Pisagua y la distribución de los cementerios.

\section{BIBLIOGRAFIA}

ALBORNOZ ACOSTA, I.A. Antecedentes sobre estudios de 1977 suelo y agua del sector de Isluga. Altiplano Tarapaqueño, Centro Isluga Investigaciones Andinas, Universidad del Norte, Iquique, Chile.

ANDERSON, J.E. The human skeleton. A manual for 1962 archaeologist, National Museum of Canada, Otawa.

BAFFI, E.I. 1989

BASS,W.M. 1971

BENFER, R.A. 1984

Antropología física de la región valliserrana. III. Relaciones Biológicas con Selvas Occidentales, Informe Beca Perfeccionamiento CONICET, Buenos Aires.

Human osteology: A laboratory and field manual of the human skeleton, University of Missouri Press, Springfield. tism: The preceramic village of paloma, Peru, en Paleopathology at the origins of Agriculture, Ed. M. N. Cohen y G. J. Armelagos, pp. 531-558, Academic Press.

BENNETT, K.A. On the estimation of some demogra1973 phic characteristics on a prehistoric populations from the american southwest, Am. J. Phys. Anthrop., 39:223-232, Philadelphia.

BERRY, A.C. y R. J. BERRY Epigenetic variation in the human 1967 cranium, J. of Anatomy, 101:361-379.

BIRD, J.

Excavations in northern Chile, Anhrop., Papers Amer. Mus. Nat. Hist., 38: 172-316, New York.

1946 The cultural sequence in the north chilean coast, Handbook of South
American Indians, 2: 587-594, Smithsonian Institution, Washington.

BLISS, C.I. Statistic in biology, Ed. Mc Graw-Hill 1967 Book, New York.

BÓRMIDA, M. Los antiguos patagones. Estudio de 1953-54 craneología, Runa 6(1-2):1-96, Buenos Aires.

1961-63 Los esqueletos de Lauricocha, Acta Prehistorica 5-7:1-34, CAEA, Buenos Aires.

BOROVANSKY, L. Sex differences in the human skull, am. 1936 Jour. Phys. Anthrop., 22:162-163. Philadelphia.

BOYD, J.D. y J.C. TREVOR Problems in reconstruction. 1953 race. sex and stature from skeletal material, pp. 133-152, en Modern trends in forensic medicine, ed. por C.K. Simpson, Butterwoth, London.

BROTHWELL, D.R. Digging-up bones, Cornell University 1981 Press, Ithaca.

BUIKSTRA,J.E. Hopewell in the lower Illinois Valley: 1976 a regional study of human biological Vvariability and prehistoric mortuary behavior, Northwestern Univ. Archaeol. Program, Sci.Pap, Illinois.

CASTRO, M. Y S. QUEVEDO Proposiciones metodológicas 1983-84 para el estudio de los rasgos no-métricos en el cráneo humano, Bol. Mus. Nac. Hist. Nat., 40:173-210, Santiago.

COCILOVO, J.A. Dimorfismo sexual y deformación cra1973 neana artificial en patagones de chubut, Actas II Simposio Int. Cs. Morfológicas, pp. 633-642, Córdoba.

1975 Estudio de dos factores que influencian 
la morfología craneana en una colección andina: el sexo y la deformación artificial, Rev. Inst. Antrop., 3(2): 197-212, Fac. Fil. Let., Univ. Nac. Tucumán.

Estudio de dos factores que influyen en la morfología craneana en una colección patagónica: el sexo y la deformación artificial, Archivos de Anatomía e Antropología, 3(3): 113-141, Río de Janeiro.

Estudio sobre discriminación y clasificación de poblaciones prehispánicas del N.O. argentino, Publ. Ocas. 36, Mus. Nac. Hist. Nat., Santiago, Chile.

Biología de la población prehistórica de Pisagua. Continuidad y cambio biocultural en el norte de Chile,Tesis Doctoral, Universidad nacional de Córdoba, Córdoba, Argentina

COCILOVO, J.A. y M. A. BORDACH. Antropología física 1985 de la Quebrada de Humahuaca. La colección Pukará de Tilcara. Estudio osteológico, Informe CONICET, Río Cuarto, Argentina.

COCILOVO, J. A. y J. DI RIENZO, Un modelo biológico 1985 para el estudio del poblamiento prehispánico del territorio argentino. Correlación fenética- espacial, Rel. Soc. Arg. Antrop., 16:119-135, Buenos Aires.

COCILOVO, J.A., F. ROTHHAMMER, S. QUEVEDO y E. 1982 LLOP, Microevolución en poblaciones prehistóricas del area andina. III. La población del Morro de Arica. Craneometría, Rev. UNRC 2(2):91-111, Río Cuarto.

COCILOVO, J.A. y F. ROTHHAMMER. Paleopopulation 1990 biology of the Southern Andes: Craniofacial chronological and geographical differentiation, Homo 41(1): 16-31, G.F. Verlag, Stuttgart-New York.

COMAS, J. Manual de Antropología Física, Univ. 1966 Nac. Aut. México, México.

CONVENCIÓN INTERNACIONAL DE MÓNACO. Unifi1906 cación de las medidas craneométricas y cefalométricas, en J. Comas 1966 Manual de Antropología Física, pp. 634641, UNAM, México.

COSTA J. M.A. Quitor 6: Sector Tardío. Memoria 1985 para optar al título de Arqueólogo, Departamento de Arqueología, Universidad del Norte, Antofagasta, Chile.

DAUELSBERG, H.P. Excavaciones arqueológicas en Quiani 1974 (Provincia de Tarapacá, Dept. Arica). Chungará, 4:7-38, Departamento de Antropología, Universidad del Norte, Arica, Chile.

DAVID, H.A., O. HARTLEY y E.E. PEARSON, The Distri1954 bution of the ratio in a single normal sample of range to standard deviation, Biometrika, 41(3-4):482-497, London.
DEMBO, A. y J. IMBELLONI. Deformaciones Iintencionales 1938 del cuerpo humano de carácter étnico, Humanior A, III, Ed. Anesi, Buenos Aires.

ERRAZURIZ, A.M., J.I. GONZÁLEZ, M. HENRIQUEZ, P. 1987 CERECEDA, M. GONZÁLEZ y R. RIOSECO. Manual de Geografía de Chile, Ed. A.Bello, Santiago, Chile.

FEREMBACH, D., I. SCHWIDETZKY y M. SLOUTKAL. 1979 Recommandations pour determiner l'Age et le sexe sur le squelette, Bull. et Mém. de la Soc. d'Anthrop. de París, 6(13):7-45, París.

FINNEGAN, M. y A. MARCSIK A non-metric examination 1979 of the relationships between osteological remains from Hungary representing populations of Avar Period, Acta Biologica Szeged, 25 (1-2): 97-118.

GENOVÉS, S.T Introducción al diagnóstico de la e1962 dad y del sexo en restos óseos prehistóricos. UNAM, México.

HRDLIÇKA, A. Practical Anthropometry. 3a ed., The Wistar Institute of Anatomy and Biology, Philadelphia.

IMBELLONI, J. Deformaciones intencionales del crá1925 neo en Sud América. Rev. Mus. La Plata, 18:328-407, La Plata.

1938 Tabla clasificatoria de los indios. Regiones biológicas y grupos raciales humanos de América. PHYSIS, 12:229-249, Buenos Aires.

1958 Nouveaux apports á la clasification de l'Homme Americain. Miscellanea Paul Rivet Octogenario Dicata, XXXI Congreso Interamericano, Universidad Autónoma de México, 1:107-136, México.

KAMPS, J.E. Paleodemografía del cementerio de Ca1984 marones 14, en V. Schiappacasse y $\mathrm{H}$. Niemeyer "Descripción y Análisis Interpretativo de un Sitio Arcaico Temprano en la Quebrada de Camarones, Publ. Ocas. 41, Mus. Nac. Hist. Nat., pp. 163-172, Santiago.

KEEN, J.A. A Study of the differences between ma1950 le and female skulls, Am. Jour. Phys. Anthrop., 8:65-79, Philadelphia.

LANINO R., I.M. Antecedentes de las explotaciones 1977 agrícolas en Isluga. Altiplano de la Provincia de Iquique, Centro Isluga de Investigaciones Andinas, Universidad del Norte, Iquique, Chile.

LARNACH, S.L. y L. FREEDMAN, Sex determination of 1964 aboriginal crania from coastal New South Wales, Australia. Records of the Australian Museum, 26(11): 295-308, Sydney.

LISON, L. Estadística Aplicada a la Biología Ex1976 perimental. Ed. EUDEBA, Buenos Aires.

MARTINO, A.L. y J.A. COCILOVO. El uso y la importancia 
1988

de los atributos morfológicos. II Comparación de dos grupos de la Costa Norte de Chile (Morro de Arica y Pisagua). Estudios de Antropología Biológica, $\mathrm{V}$ Congreso de Antropología Física Juan Comas: pp. 151-175, México.

MARTINO, A.L., M. CASTRO y S. QUEVEDO, La distribu1991 ción de atributos morfológicos según el sexo, la edad y la deformación artificial de la población Morro de Arica (3000 aC), Antropología Biológica, ALAB 1(1): 33-48, Santiago, Chile.

MENDONÇA, O.J. y J.A. DI RIENZO, La deformación cra1981-82 neana artificial en la serie masculina de Morro de Arica (Chile), Segunda Parte, Rel. Soc. Arg. Antrop., 14(2):49-66, Buenos Aires.

MENDONÇA, O.J., J. DI RIENZO y M.A. BORDACH 1983 La deformación craneana artificial en la serie masculina de Morro de Arica (Chile). Primera Parte, Rev. UNRC., 3(1):27-40, Río Cuarto.

MENDONÇA, O.J., M.A. BORDACH y J.A. DI RIENZO

1986 La deformación craneana artificial en la serie femenina de Morro de Arica (Chile), Primera Parte, Runa 16:85-102, Buenos Aires.

MOSTNY, G. Prehistoria de Chile, Editorial Univer1977 sitaria, Santiago.

MUNIZAGA, J.R. Deformación cefálica intencional (aná1964 a lisis de algunas poblaciones precolombinas en el Norte de Chile), Antropología 2(2):5-18, Centro de Estudios Antropológicos, Universidad de Chile, Santiago, Chile.

1964 b Comparación de poblaciones precolombinas del norte de Chile (empleo de rasgos morfológicos de variación discontinua), Antropología 2:87-95, Centro de Estudios Antropológicos, Santiago.

1974 Deformación craneal y momificación en Chile, Anales de Antropología, 11:329336, México.

1976 Paleoindio en sudamérica (restos óseos humanos de las cuevas de Palli Aike y Cerro Sota, Pcia. de Magallanes, Chile). Homenaje al Dr. G. Le Paige, pp. 19-30, Universidad del Norte, Chile.

NEUMAN, G.K. Types of artificial cranial deformation 1942 in the Eastern United States, American Antiquity, 7:306-310, U.S.A.

NUÑEZ,L.A. 1965

1968
Desarrollo cultural prehispánico del norte de Chile. Estudios Arqueológicos 1:37-109, Universidad de Chile, Antofagasta.

Subárea Loa-Costa chilena desde Copiapó a Pisagua, Región Norte Grande de Chile, Zona: Río Loa-Río Camiña (De cordillera a mar), Actas y Memorias 37 Cong. Int. Amer., 2:145-180, Buenos Aires.

Northern Chile, en R.E. Taylor y C.W.
Meighan, Chronologies in New World Archaeology, pp. 483-511, Ed. Academic Press, New York.

1980 Cazadores tempranos en andes meridionales, Bol. Antrop. Amer.,2:87-120, Instituto Panamericano de Geografía e Historia.

NUÑEZ L.A. y J. VARELA. Sobre los recursos de agua y el 1967-68 poblamiento de la costa del Norte Grande de Chile, Estudios Arqueológicos, 3-4:7-16, Universidad de Chile, Antofagasta, Chile.

NUÑEZ, L.A.. V. ZLATAR y P. NUÑEZ. Un circuito trans1976 humántico entre la costa de Pisagua y el borde occidental de la Pampa del Tamarugal, Estudios Atacameños, 3:49-52, San Pedro de Atacama.

ORELlANA, M. La cultura San Pedro. Arqueología Chi1963 lena, 3(17), Centro de estudios Antropológicos, Universidad de Chile, Santiago.

OSSEMBERG, N.S. The influence of artificial cranial on 1970 discontinuous morphological traits. A. J. Phys. Anthrop., 33:357-371, Philadelphia.

1987 Retromolar foramen of the human mandible. Am. J. Phys. Anthrop., 73:119-128, Philadelphia.

PERIZONIUS, W. R. K. Non-metric Cranial traits: sex diffe1979 rences and age dependence, J. of Hum. Evol., 8:679-684.

PUCCIARELLI. H. M. Relaciones entre huesos wormianos y 1972 otros rasgos neurocraneanos sobre un grupo racial homogéneo. An. Soc. Cient. Arg., 194:233-243, Buenos Aires.

1973 Influence du milieu dans la formation des os wormiens chez des groupes raciaux Sud-Américains, Biometrie humaine, 8:53-62, París.

QUEVEDO, S. Estudio de un cementerio pre-histó1976 rico exploración de sus potencialidades demográficas y socio-culturales. Tesis de Lic. en Arqueología y Prehistoria, Fac. Cs. Humanas, Univ. Chile, Santiago.

QUEVEDO, S. y J. A. COCILOVO, La población antigua de 1996 Pisagua. Caracterización bioantropológica del cementerio " $\mathrm{C}$ " de la colección Max Uhle, Informes DIBAM 4:46-53, Santiago, Chile.

1995 El cementerio Protonazca de Pisagua ("D" colección Max Uhle 1913), Caracterización biocultural. Hombre y Desierto. Una Perspectiva Cultural, Instituto de Investigaciones Antropológicas, Universidad de Antofagasta, Sociedad Chilena de Arqueología, Antofagasta, Chile.

RAO, C. R. Advanced statistical methods in bio1952 metrics Research,J. Wiley, N. York. USA. 
RIVERA, M. Una hipótesis sobre movimientos pobla1975 cionales altiplánicos y transaltiplánicos en las costas del norte de Chile. Chungará, 5:7-32, Departamento de Antropología, Universidad de Tarapacá, Arica.

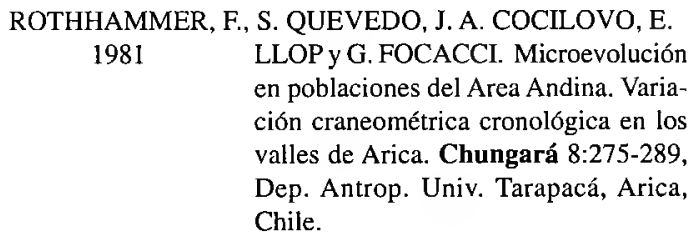

ROTHHAMMER, F., S. QUEVEDO, J. A. COCILOVO y E. 1982 LLOP. Microevolution in Prehistoric andean population. Chronologic craniometric variations. Am. J. Phys. Anthrop. 58(4):391-396,Philadeiphia.

1984 Microevolution in prehistoric andean population. Chronologic cranio-metric non-metrical Variation, Am. J. Phys. Anthrop., 65(2):157-162, Philadelphia.

1983 Afīnidad biológica de las poblaciones prehistóricas del litoral ariqueño con grupos poblacionales costeros peruanos y altiplánicos. Chungará 11: 161-165, Dto. Antrop. Univ. Tarapacá, Arica, Chile.

ROTHHAMMER, F., J. A. COCILOVO y S. QUEVEDO. El 1984 poblamiento temprano de Sudamérica, Chungará, 13:99-108, Univ. Tarapacá, Arica, Chile.

1986 Origen y microevolución de la población chilena. En Culturas de Chile, Prehistoria, Desde sus Orígenes hasta los Albores de la Conquista (varios autores), pp. 403-413, Ed. Andrés Bello, Santiago, Chile.

ROTHHAMMER, F. y M. RIVERA. Evaluación biologica y 1986 cultural de poblaciones Chinchorro: Nuevos elementos para la hipótesis de contactos transaltiplánicos, cuenca Amazonas-Costa Pacífico, Chungará 16-17: 295-306, Arica, Chile.

SHAPIRO, S. S. y M. B. WILK. An Analysis of variance test 1965 for normality (complete samples). Biometrika, 52(3-4): 591-611, London.

SCHEFFÉ, $H$. The Analysis of variance, Ed. J. Wiley 1959 and Sons, New York.

SEBER G.A.F Multivariate observation, J. Wiley 1984 and Sons, USA.

SNEATH, P. H. A. y R. R. SOKAL. Numerical Taxonomy W. 1973 H. Freeman and Comp., San Francisco.
SNEDECOR, G. W. y W. G. COCHRAN, Métodos estadís1984 ticos, Ed. CECSA, México.

SOKAL, R. R. y F. J. ROHLF, Biometría. Ed. Blume, Ma1979 drid, España

STEWART, T.D. Essential of forensic anthropology, 1979 Ed. Ch. Thomas Publ., Springfield.

SULLIVAN, L.R. The frecuency and distribution of some 1922 anatomical variations in american Crania. Anthrop. Papers, Am. Mus. of Nat. Hist., 23:203-258.

TESTUT, $L$. Tratado de anatomía humana, $\mathrm{t}$ 1, Ed. 1921 Salvat, Barcelona.

UBELAKER, D.H. Reconstruction of Demographic Profiles 1974 from Ossuary Skeletal Samples. A Case Study from the Tidewater Potomac. Smithsonian contributions to anthropology, 18, pp.1-79. Washington.

UHLE, M. Ausgrabungen im nördlichen Chile,

1913 Zeitschrift für Ethnologie, 45, Jahrgang, Heft 6, pp. 1141-1142, Berlin.

1919 La Arqueología de Arica y Tacna. Bol. Soc. Ecuat. Est. Hist. Amer., Quito.

VARELA, H.H. La Población prehistórica de San Pe1997 dro de Atacama. Composición, estructura y relaciones biológicas, Tesis Doctoral, Universidad Nacional de Río Cuarto, Río Cuarto, Argentina.

VIVES, E. y J. BERTRANDPETIT. Les non-métricques et 1986 descripties du crane déformé. Bull. et Mém. Soc. d'Anthrop. París, 3,14 (4):237-248.

WEISS, $\mathrm{P}$. 1961

Osteología Cultural. Prácticas Cefálicas, 2a Parte, Lima.

1962 Tipología de las deformaciones cefálicas. Rev. Mus. Nac., 31:15-42, Lima.

WILDER, H. H. Laboratory Mannual of Anthropo1920 metry. P. Blakiston's Son and Co., Philadelphia. 
Apéndice 1:

Atributos gruesos y sus categorias

\begin{tabular}{|c|c|c|c|c|}
\hline $\mathrm{N}^{\mathbf{0}}$ & Denominación del atributo & \multicolumn{3}{|c|}{ Categorías de observación } \\
\hline 1 & Conservacion & Bueno & Regular & Malo \\
\hline 2 & Tamaño & Pequeño & Mediano & Grande \\
\hline 3 & Peso & Liviano & Mediano & Pesado \\
\hline 4 & Relieves oseos & Suaves & Regulares & Fuertes \\
\hline 5 & Forma de la cara & Angosta & & Ancha \\
\hline 6 & Glabela & Suave & & Prominente \\
\hline 7 & Tamaño arcos superciliares & Suaves & Medianos & Marcados \\
\hline 8 & Forma de las orbitas & Redondas & Intermedias & Cuadradas \\
\hline 9 & Abertura fosas nasales & Pequeña & Mediana & Grande \\
\hline 10 & Espina nasal & Reducida & & Marcada \\
\hline 11 & Tamaño huesos malares & Pequeños & Medianos & Grandes \\
\hline 12 & Desgaste dentario & Leve & Mediano & Pronunciado \\
\hline 13 & Vertex & Suave & Medio & Pronunciado \\
\hline 14 & Inclinacion frontal & Suave & Mediana & Fuerte \\
\hline 15 & Surco retroglabelar & Suave & & Marcado \\
\hline 16 & Prominencia huesos nasales & Leve & & Pronunciada \\
\hline 17 & Arcos cigomaticos & Gráciles & Medios & Robustos \\
\hline 18 & Cresta supramastoidea & Suave & Mediana & Pronunciada \\
\hline 19 & Occipucio & Suave & Medio & Marcado \\
\hline 20 & Apofïsis mastoideas & Pequeñas & Medianas & Grandes \\
\hline 21 & Altura boveda craneana & Baja & Mediana & Alta \\
\hline 22 & Relieves plano nucal & Suave & Medianos & Marcados \\
\hline 23 & Protuberancia occipital externa & Suave & Media & Marcada \\
\hline 24 & Perfil craneano & \multicolumn{3}{|c|}{$\begin{array}{l}\text { Ovoide, Elipsoide, Pentagonoide, Esfenoide, Esferoide, } \\
\text { Romboide. Brisoide }\end{array}$} \\
\hline 25 & Arco cigomatico & Criptocigia & & Fenocigia \\
\hline 26 & Plagiocefalia & Presencia & & Ausencia \\
\hline 27 & Forma foramen magnum & Circular & & Poligonal \\
\hline 28 & Depresión del esfenoides & Leve & Mediana & Pronunciada \\
\hline 29 & Tamaño fosas glenoideas & Pequeñas & Regulares & Grandes \\
\hline
\end{tabular}

